\title{
Optimal 2D auxetic micro-structures with band gap
}

\author{
Matteo Bruggi, Alberto Corigliano \\ Department of Civil and Environmental Engineering, Politecnico di Milano, I20133, Milano, Italy
}

\begin{abstract}
A systematic investigation is presented that explores band gap properties of periodic microstructures architected for maximum auxeticity. The design of two-dimensional auxetic cells is addressed using inverse homogenization. A non-convex optimization problem is formulated that is solved through mathematical programming. Different starting guesses are used to explore local minima when distributing material and void or two materials and void. The same numerical tool succeeds in capturing re-entrant, chiral and anti-chiral layouts with negative Poisson's ratio, retrieving solutions originally found through other approaches as well as generating variations. A Floquet-Bloch approach is then applied to the achieved periodic cells to investigate possible band gaps characterizing the in-plane wave propagation. Directional and full band-pass filters are found in the case of micro-structures whose auxetic behavior comes from the arising of a rotational deformation of the periodic cell. Such kind of topologies could be exploited to design tunable wave guides and tunable phononic crystals, respectively.
\end{abstract}

Keywords: inverse homogenization, material design, auxetic metamaterials, phononic band gap, chirality.

Email addresses: matteo.bruggi@polimi.it (Matteo Bruggi), alberto.corigliano@polimi.it (Alberto Corigliano) 


\section{Introduction}

Metamaterials are endowed with effective properties that are generated from their bulk behavior or their internal structuring and are not found in naturally occurring materials, see e.g. [1]. The most popular feature of auxetic metamaterials is that they expand in the direction perpendicular to an externally exerted tension. Also, auxetic metamaterials are of interest because of the enhanced properties related to their negative Poisson's ratio, such as increased shear resistance [2], indentation resistance [3], fracture toughness [4], synclastic behaviour [5], variable permeability [6] and energy absorption [7].

The work in [5] firstly reported a re-entrant foam structure which exhibits negative Poisson's ratio, whereas the term auxetic was coined after the investigation on molecular network design in [8]. The recent work by [9] provides an extensive review on auxetic metamaterials, including a classification of the structural and cellular shapes that exhibit such a counter-intuitive deformation behavior. Rotating polygonal models, see e.g. the rhombi and parallelograms systems in [10], and crumpled or perforated sheets models, see e.g. the graphene sheets with defects in [11] or the porous isotropic material in $[12,13]$, provide negative Poisson's ratio. Alternatively, auxetic microstructures can be synthesized exploiting re-entrant honeycombs or lattices with chiral geometrical properties, see e.g [14] and [15], respectively.

In general, periodic micro-structures feature unique wave propagation characteristics, as the capability of attenuating elastic waves over certain frequency bands, the so-called stop bands or band gaps, see in particular [16]. The arising and the extension of the possible band gaps mainly depend on the topology of the representative unit cell, whereas its central frequencies strongly depend on the geometry and on the material(s) employed in the periodic micro-structure. These unique filtering capabilities can be exploited to synthesise micro-structures with periodically varying properties for vibration isolation or wave-guiding purposes. Band gap characteristics of two-dimensional periodic micro-structures have been extensively investigated e.g. in [17-19], 
whereas a limited number of works focus on stop bands in auxetic micro-structures. Among them, [20] compares re-entrant topologies to hexagonal honeycombs, [21] investigates hierarchical auxetic rectangular perforated composite metamaterials, whereas [22] combines hexachiral lattices with local resonators. Reference is also made to $[23,24]$ for effective applications of 3D microlattices as mechanical filters and also with auxetic properties [25, 26].

Topology optimization [27] was firstly employed as a material design tool by [28] that formulated an inverse homogenization approach to tailor optimal micro-structures with prescribed mechanical properties using truss or frame elements. Later on, the classical formulation for the maximization of a target performance distributing a given amount of material in the design domain was implemented to synthesize optimal period micro-structures, including auxetic composites, see e.g. [29]. Since then, many works have addressed the topic of topology optimization for architected materials design, as recently reviewed by [30]. Two-dimensional auxetic micro-structures have been investigated, among the others, by [31, 32] using a level-set based optimization method, [33] implementing topological derivatives and [34] resorting to the synthesis of compliant mechanisms and to a micropolar material model. The work in [35] provides an efficient implementation of an energy-based homogenization approach coupled to an optimality criterion, [36] implements a robust formulation against the arising of local "hinges", [37] adopts nonlinear modelling to cope with large deformations. The design of auxetic micro-structures made of multiple materials can be found e.g. in [38, 39], whereas three-dimensional lattices are dealt with e.g. in [36, 40, 41].

As discussed in [29] and [35], the optimal design of materials with extreme elastic properties allows for multiple local minima. Within the above framework, the synthesis of two-dimensional auxetic periodic micro-structures is performed using an inverse energy-based homogenization approach with the Solid Isotropic material Model with Penalization (SIMP) [27] and the Rational Approximation of Material Properties (RAMP) [42]. An efficient volume-constrained formulation is implemented along with a density-based filtering scheme. The arising optimization problem is 
solved by means of sequential convex programming [43], distributing material and void with SIMP, or two materials and void with SIMP and RAMP. A Floquet-Bloch approach is then applied to the achieved periodic micro-structures to investigate in-plane wave propagation, see e.g. [44].

As shown in [25], the auxetic feature can be conveniently exploited to tune band gap properties of phononic crystals. When an external tensile/compressive load is applied to the micro-structure, the auxetic unit cell changes its configurations through streching/shrinking strains along all the directions. This preserves the topology of the cell while resulting in the tuning, either hardening or softening, of the frequencies of the modes limiting band gaps.

This contribution provides a systematic investigation that explores band gap properties of periodic micro-structures architected for maximum auxeticity. Enhanced band gap tuning in negative Poisson's ratio materials and optimization to control both auxeticity and band gap remain to be demonstrated. This work may be regarded only as a first exploration in this direction. The achieved material layouts are classified depending on the deformation that gives the extreme auxetic behavior and, based on the partial and full band gaps detected in the analysis of the cells, recurring trends are outlined for each class. Indeed, such an investigation allows identifying types of micro-structures that could be effectively exploited to synthesize tunable phononic crystals (where full band gaps prevent propagation independently of the wave direction) and tunable wave guides (where partial band gaps prevent propagation only for selected wave directions).

It must be remarked that, adopting different starting guesses, the same formulation for maximum auxeticity retrieves re-entrant, chiral and anti-chiral layouts with negative Poisson's ratio. Topologies with different auxetic deformations are detected by the same numerical tool since they arise as local minima of the one-parameter-dependent objective function using filtered densities as unknowns. Optimal layouts that were originally revealed through the adoption of different formulations, modelling assumptions and relaxations of the continuous problem of material design, can be retrieved by the implemented approach, see in particular results among the chiral and anti- 
chiral tessellations of Sections 4.2 and 4.3. The same algorithm can generate alternative solutions handling single-material and bi-material cells. In particular, Section 4.1 shows variations of reentrant auxetic structures, whereas Section 4.4 addresses optimal auxetic micro-structures where one of the materials acts as a coating for the other, as well as layouts where two sub-structures work in parallel for maximum auxeticity.

The systematic investigation on wave propagation points out that directional and full bandpass filters mainly arise in the case of optimal cells whose auxetic behavior comes from a rotational deformation of the micro-structure. Volume fraction and material properties are shown to remarkably affect the amplitude and the central frequency of the possible directional/full band gaps. Modes limiting full band gaps are analyzed both in case of single-material and bi-material cells.

The layout of the paper is as follows. The mathematical formulation governing the inverse homogenization problem for the optimal design of periodic micro-structures is presented in Section 2 , along with details on the discrete problem and on the solution strategy. The in-plane wave propagation in periodic micro-structures is addressed in Section 3 and in Appendix A. Section 4 reports the outcome of the numerical simulations, whereas Section 5 summarizes the main results and discusses perspectives of the work described in this paper.

\section{Homogenization and optimal design of periodic micro-structures}

A classical way to the optimal design of periodic microstructures through the inverse homogenization approach [28] is based on the adoption of the asymptotic homogenization method, see e.g. $[45,46]$. Within a three-dimensional framework, $z_{1}, z_{2}, z_{3}$ are the macroscopic coordinates, whereas $y_{1}, y_{2}, y_{3}$ are the microscopic coordinates. A single base cell $Y$ is considered with dimension $\bar{y}_{1}, \bar{y}_{2}$, $\bar{y}_{3}$. Denoting by $\epsilon$ the aspect ratio between the macro scale and the micro scale, the macroscopic 
displacement can be written as:

$$
\mathbf{u}^{\epsilon}(\mathbf{z})=\mathbf{u}_{0}(\mathbf{z}, \mathbf{y})+\epsilon \mathbf{u}_{1}(\mathbf{z}, \mathbf{y})+\epsilon^{2} \mathbf{u}_{2}(\mathbf{z}, \mathbf{y})+\ldots, \mathbf{y}=\mathbf{z} / \epsilon
$$

When $\epsilon \ll 1$, the dependence on $\mathbf{y}$ can be treated as periodic for any fixed macroscopic point with coordinate $\mathbf{z}$. Considering only the first order terms in the asymptotic expansion of Eqn. (1), the homogenized stiffness tensor $E_{i j k l}^{H}$ can be found as:

$$
E_{i j k l}^{H}=\frac{1}{|Y|} \int_{Y} C_{p q r s}\left(\varepsilon_{p q}^{0(i j)}-\varepsilon_{p q}^{(i j)}\right)\left(\varepsilon_{r s}^{0(k l)}-\varepsilon_{r s}^{(k l)}\right) d Y
$$

where $|Y|$ is the volume of the base cell, $C_{p q r s}$ is the local stiffness tensor, $\varepsilon_{p q}^{0(i j)}$ are prescribed macroscopic strain fields. The microscopic strain fields $\varepsilon_{r s}^{(k l)}=\varepsilon_{r s}\left(\chi^{k l}\right)$ come from the Y-periodic solution of the micro scale problem:

$$
\int_{Y} C_{p q r s} \varepsilon_{p q}(v) \varepsilon_{r s}\left(\chi^{k l}\right) d Y=\int_{Y} C_{p q r s} \varepsilon_{p q}(v) \varepsilon_{r s}^{0(k l)} d Y, \quad \forall v \in H^{1}(Y)
$$

where $\chi$ is the microscopic displacement field and $v$ is the $Y$-periodic test function. Instead of the asymptotic method, average stress and strain theorems can be adopted to implement an equivalent energy-based approach, see [35] among the others. Accordingly, unit test strains are enforced directly at the boundaries of the unit cell to induce $\varepsilon_{p q}^{A(i j)}=\varepsilon_{p q}^{0(i j)}-\varepsilon_{p q}^{i j}$, and Eqn. becomes:

$$
E_{i j k l}^{H}=\frac{1}{|Y|} \int_{Y} C_{p q r s} \varepsilon_{p q}^{A(i j)} \varepsilon_{r s}^{A(k l)} d Y .
$$

In 2D, prescribed macroscopic strain fields are three independent unit strain fields, i.e. the unit strain in the horizontal direction $(11 \rightarrow 1)$, the unit strain in the vertical direction $(22 \rightarrow 2)$ and the unit shear strain $(12=21 \rightarrow 3)$, the homogenized elasticity tensor may be written in matrix 
form as:

$$
\mathbf{E}^{H}=\left[\begin{array}{ccc}
E_{11}^{H} & E_{12}^{H} & E_{13}^{H} \\
& E_{22}^{H} & E_{23}^{H} \\
& & E_{33}^{H}
\end{array}\right],
$$

and the constitutive matrix of the constituent isotropic material reads:

$$
\mathbf{C}=\left[\begin{array}{ccc}
\lambda+2 \mu & \lambda & 0 \\
\lambda & \lambda+2 \mu & 0 \\
0 & 0 & \mu
\end{array}\right]
$$

where $\lambda$ and $\mu$ are the Lamé constants of the medium.

This work deals with periodic "material and void" or "two-materials and void" micro-structures. In both cases, a maximum fraction $V_{f}$ of "full material" is allowed, whereas the remaining part of the domain, i.e. the fraction $1-V_{f}$, consists of "void". In the "two-materials and void" case, the full material region reads $V_{f}=V_{h}+V_{s}$, where $V_{h}$ stands for the maximum admissible fraction of hard material and $V_{s}$ for the fraction of soft material. As a simplification, it is assumed that both materials share the same Poisson's ratio. The point-wise interpolation of the properties of the isotropic constituent material is based on a double discretization of the field of the minimization unknowns. Discretizing the base cell with $N$ four-node square finite elements, two groups of $N$ unknowns are considered, namely $\mathbf{x}$ and $\mathbf{x}_{m}$. In the $e$-th element, $x_{e}$ is used in the framework of a classic SIMP (Solid Isotropic Material with Penalization) to interpolate between the case where there is material $\left(x_{e}=1\right)$ or not $\left(x_{e}=x_{m i n}\right)$, whereas $x_{m, e}$ enters a RAMP (Rational Approximation of Material Properties) to model the variation between hard $\left(x_{m, e}=1\right)$ and soft material $\left(x_{m, e}=0\right)$, see $[27,42]$. The resulting interpolation of the constitutive matrix in the $e$-th element therefore reads:

$$
\mathbf{C}_{\mathbf{e}}=x_{e}^{p}\left(\mathbf{C}_{\mathbf{s}}+\frac{x_{m, e}}{1+q\left(1-x_{m, e}\right)}\left(\mathbf{C}_{\mathbf{h}}-\mathbf{C}_{\mathbf{s}}\right)\right),
$$


where $\mathbf{C}_{\mathbf{h}}$ and $\mathbf{C}_{\mathbf{s}}$ are the constitutive matrices of the hard and soft isotropic materials, respectively, whereas $p=q=3$ in the computations. Eqn. (7) is ideally conceived to penalize intermediate densities and converge to pure $0-1$ solutions. For $\mathbf{C}_{\mathbf{h}}=\mathbf{C}_{\mathbf{s}}$, it handles periodic "material and void" micro-structures with no modification. It is assumed that $x_{\min }=10^{-3}$ to avoid any potential singularity of the global stiffness matrix in the finite element discretization.

The computation of the generic component of the homogenized elasticity matrix involves element mutual energies that may be computed exploiting classic algorithms for topology optimization:

$$
E_{i j}^{H}=\frac{1}{|Y|} \sum_{e}^{N}\left(\mathbf{U}_{e}^{A(i)}\right)^{T} \mathbf{K}_{0, e}\left(x_{e}, x_{m, e}\right) \mathbf{U}_{e}^{A(j)}
$$

where $\mathbf{U}_{e}^{A(i)}$ is the displacement vector of the $e$-th element corresponding to the enforcement of the unit strain field along the direction $y_{1} \varepsilon^{0(11)} \rightarrow \varepsilon^{0(1)}$, the unit strain field along the direction $y_{2} \varepsilon^{0(22)} \rightarrow \varepsilon^{0(2)}$ and the unit shear strain $\varepsilon^{0(12)} \rightarrow \varepsilon^{0(3)}$, and $\mathbf{K}_{0, e}\left(x_{e}, x_{m, e}\right)$ is the stiffness matrix of the element that depends on the minimization unknowns according to Eqn. (7). Defining by $\mathbf{N}$ the matrix of the shape functions, $\mathbf{B}=\boldsymbol{\partial} \mathbf{N}$ the strain-displacement matrix in the small strains and displacements hypothesis and $\Omega_{e}$ the element volume, it reads:

$$
\mathbf{K}_{0, e}=\int_{\Omega_{e}} \mathbf{B}^{T} \mathbf{C}_{\mathbf{e}}\left(x_{e}, x_{m, e}\right) \mathbf{B} d \Omega_{e}
$$

Hence, a generic problem of material design for a discretized finite element model with $N$ elements that aims at synthesizing periodic "material and void" or "two-material and void" micro- 
structures reads:

$$
\left\{\begin{aligned}
\min _{\substack{x_{m i n} \leq x_{e} \leq 1 \\
0 \leq x_{m, e} \leq 1}} f\left(E_{i j}^{H}\left(\mathbf{U}^{A(i)}, \mathbf{U}^{A(j)}\right)\right) \\
\text { s.t. }\left[\sum_{e}^{N} \mathbf{K}_{0, e}\left(x_{e}, x_{m, e}\right)\right] \mathbf{U}^{A(i)}=\mathbf{F}^{A(i)} \quad i=1,2,3, \\
f\left(E_{i j}^{H}\right) \leq \bar{f} \\
\frac{1}{V} \sum_{N} x_{e} V_{e} \leq V_{f} \\
\frac{1}{V} \sum_{N} x_{e} x_{m, e} V_{e} \leq V_{h}
\end{aligned}\right.
$$

the summation in Eqn. (10b) symbolizes the assembling of stiffness matrices of the $N$ elements. In the above relations, the objective function includes components of the homogenized elasticity matrix to synthesize materials with peculiar properties. The first constraint, i.e. Eqn. (10b), enforces the unit strain fields to compute mutual energies, being $\mathbf{U}^{A(i)}$ and $\mathbf{F}^{A(i)}$ the global displacement vector and the right hand side vector referring to $\varepsilon^{0(i)}$. Eqn. (10c) is a general inequality enforcement introducing some bound on a combination of the components of the homogenized elasticity matrix. The third constraint enforces the allowable volume fraction $V_{f}$ of material, being

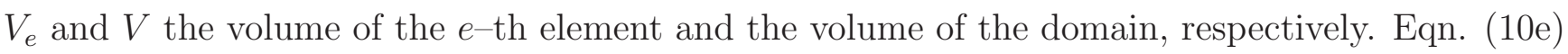
prescribes the maximum amount of hard material $V_{h}$. This constraint is inactive when periodic "material and void" micro-structures are sought for $\mathbf{C}_{\mathbf{h}}=\mathbf{C}_{\mathbf{s}}$.

Periodic boundary conditions on the base cell $Y$ are imposed in a direct manner, meaning that redundant unknowns are eliminated when handling Eqn. (10b). Following [35], $\mathbf{U}^{A(i)}$ is divided in four parts: $\mathbf{U}_{1}^{A(i)}$ denotes values of prescribed displacements at the corner nodes to avoid rigid modes, $\mathbf{U}_{2}^{A(i)}$ denotes the unknowns corresponding to the interior nodes, $\mathbf{U}_{3}^{A(i)}$ and $\mathbf{U}_{4}^{A(i)}$ refer 
to unknowns corresponding to pair of nodes that are located on the opposite boundaries of the base cell satisfying $\mathbf{U}_{4}^{A(i)}=\mathbf{U}_{3}^{A(i)}+\mathbf{W}^{A(i)}$. The vector $\mathbf{W}^{A(i)}$ is populated by terms of the type $w_{p}^{i}=\varepsilon_{p q}^{0(i)} \Delta y_{q}$, where $w_{p}^{i}$ is the difference in terms of the $p$-th component of the displacement vector on a pair of nodes on opposite boundaries, being $\boldsymbol{\Delta} \boldsymbol{y}$ the difference in terms of their coordinates y. Hence, with some simplifications in the notation, Eqn. (10b) becomes:

$$
\left[\begin{array}{cc}
\mathbf{K}_{22} & \mathbf{K}_{23}+\mathbf{K}_{24} \\
\operatorname{sym} & \mathbf{K}_{33}+\mathbf{K}_{34}+\mathbf{K}_{43}+\mathbf{K}_{44}
\end{array}\right]\left[\begin{array}{c}
\mathbf{U}_{2} \\
\mathbf{U}_{3}
\end{array}\right]=-\left[\begin{array}{c}
\mathbf{K}_{21} \\
\mathbf{K}_{31}+\mathbf{K}_{41}
\end{array}\right] \overline{\mathbf{U}}_{\mathbf{1}}-\left[\begin{array}{c}
\mathbf{K}_{24} \\
\mathbf{K}_{34}+\mathbf{K}_{44}
\end{array}\right] \overline{\mathbf{W}}
$$

\subsection{Optimal design of auxetic micro-structures}

The Poisson effect is the phenomenon in which a material tends to contract in the direction perpendicular to the direction of stretching. Its measure is the ratio between the transverse strain $\left(\varepsilon_{t}\right)$ and the longitudinal strain $\left(\varepsilon_{l}\right)$ in the elastic loading direction that reads $\nu=-\varepsilon_{t} / \varepsilon_{l}$. Auxetic materials are those that extend in the direction perpendicular to the direction of stretching, exhibiting $\nu<0$, see [47]. Hence, optimal auxetic 2D micro-structures can be synthesized maximizing the ratios $\varepsilon_{22} / \varepsilon_{11}$ and $\varepsilon_{11} / \varepsilon_{22}$, that means minimizing $E_{12} / E_{22}$ and $E_{12} / E_{11}$ since negative values of $E_{12}$ are expected. These two quantities will be referred to as effective Poisson's ratios, $\nu_{12}^{*}$ and $\nu_{21}^{*}$ respectively. Indeed, $\nu_{i j}^{*}>0$ is the Poisson's ratio that corresponds to a contraction in direction $j$ when an extension is applied in direction $i$.

As shown by [28], the construction of negative Poisson's ratio materials is a challenging task. In order to achieve successful design of such kind of materials a formulation adopting an objective function of the type $f=E_{12} / E_{11}$ or $f=E_{12} / E_{22}$ should be endowed with additional constraints on isotropy or with a lower bound on the homogenized bulk modulus, see Eqn. (10c). Alternatively, the following heuristic objective function has been successfully implemented in volume-constrained problems:

$$
f=E_{12}^{H}-\beta^{s}\left(E_{11}^{H}+E_{22}^{H}\right),
$$


where $0<\beta<1$ and $s$ is the current step number during the optimization. Reference is made in particular to the original proposal by [35] and the modifications discussed in [48] for the design of "material and void" micro-structures, whereas [39] is referred for the synthesis of "two-material and void" layouts. Using the above objective function, optimal anisotropic micro-structures are expected with $\nu_{12}^{*}=\nu_{21}^{*}$. No additional constraint is enforced on the symmetry of the material base cell.

The arising optimization problem is solved via mathematical programming, adopting the Method of Moving Asymptotes (MMA) [43] as minimizer. To this purpose, at each iteration the sensitivity computation of the objective function is required with respect to the minimization unknowns $x_{e}$ and $x_{m, e}$. Because of the adjoint method, the derivatives of Eqn. (8) can be straightforwardly achieved by replacing, in the same formula, each stiffness matrix with that obtained using the derivatives of Eqn. (7).

The density filter, see e.g. [49], is implemented in the numerical simulations to sketch optimal auxetic micro-structures avoiding checkerboard and mesh-dependence at a global level (the overall topology is preserved with mesh refinement). The original design variables $x_{e}$ are simply replaced by a new set of physical unknowns $\tilde{x}_{e}$ that are computed as a weighted sum of the densities of the neighborhood elements lying within the circular domain of radius $r_{f, \min }$. In the numerical simulations, $r_{f, \min }=1.5 l$ with $l$ side of the square finite elements. No modification is applied to $x_{m, e}$. To ensure mesh-independence both at a global level and at local level (preventing the arising of small features as "hinges"), the robust approach adopted in [36] should be alternatively implemented.

\section{In-plane wave propagation in optimal periodic micro-structures}

Dealing with time-harmonic waves in the achieved periodic media, the so-called Floquet-Bloch theory is adopted, see e.g. [44, 50]. Phononic bandgap materials are generally characterized by 
their band diagram, which relates the wave vector for freely propagating waves to the frequency of propagation. The entire band diagram can be efficiently computed from analyses on the base cell if it is assumed that modes can be described by the Bloch wave expansion:

$$
\mathbf{u}(\mathbf{y}, t)=\widetilde{\mathbf{u}}(\mathbf{y}) e^{i \mathbf{k}^{T} \mathbf{y}} e^{i \omega t}
$$

where $\mathbf{y}$ is the already introduced microscopic coordinate, $t$ the temporal coordinate, $\omega$ the frequency, $\mathbf{k}$ is the wave vector, and $\widetilde{\boldsymbol{u}}$ is a $Y$-periodic wave function.

Following in particular [51], Eqn. (13) is inserted in the equation for in-plane wave propagation in a linear elastic medium. After some simplifications, one gets:

$$
\boldsymbol{\partial}_{k}^{T} \mathbf{C} \boldsymbol{\partial}_{k} \widetilde{\mathbf{u}}-\omega^{2} \rho \widetilde{\mathbf{u}}=\mathbf{0},
$$

where the differential operator reads:

$$
\boldsymbol{\partial}_{k}=\boldsymbol{\alpha}_{1}\left(\frac{\partial}{\partial y_{1}}+i k_{1}\right)+\boldsymbol{\alpha}_{2}\left(\frac{\partial}{\partial y_{2}}+i k_{2}\right), \quad \text { with } \boldsymbol{\alpha}_{1}=\left[\begin{array}{cc}
1 & 0 \\
0 & 0 \\
0 & 1
\end{array}\right], \boldsymbol{\alpha}_{2}=\left[\begin{array}{cc}
0 & 0 \\
0 & 1 \\
1 & 0
\end{array}\right]
$$

and $k_{1}$ and $k_{2}$ are the component of the wave vector $\mathbf{k}$ along $y_{1}$ and $y_{2}$, respectively.

Eqn. (14) can be solved through the finite element method. The arising equation can be converted into a linear eigenvalue problem in $\omega^{2}$ that reads:

$$
\left(\mathbf{K}\left(\mathbf{k}, \mathbf{x}, \mathbf{x}_{\mathbf{e}}\right)-\omega^{2} \mathbf{M}\left(\mathbf{x}, \mathbf{x}_{\mathbf{e}}\right)\right) \mathbf{d}=\mathbf{0}
$$

Once assigned the wave vector $\mathbf{k}$, the effective stiffness matrix is gathered from the element-wise matrices $\mathbf{K}_{\mathbf{e}}$ whose form is detailed in Appendix A. 
The mass matrix $\mathbf{M}$ comes from the assembly of the element--wise matrix:

$$
\mathbf{M}_{e}=x_{e}^{p}\left(\rho_{s}+\frac{x_{m, e}}{1+q\left(1-x_{m, e}\right)}\left(\rho_{h}-\rho_{s}\right)\right) \int_{\Omega_{e}} \mathbf{N}^{T} \mathbf{N} d \Omega_{e},
$$

with $\rho_{h}$ and $\rho_{s}$ material densities of the hard and soft component, respectively, and $\rho_{h}=\rho_{s}$ for "material and void" micro-structures.

Eqn. (16) has real and positive eigenvalues, since $\mathbf{K}$ is Hermitian and $\mathbf{M}$ is real, symmetric and positive definite. Periodic boundary conditions apply to $\mathbf{d}$, meaning that nodes on opposite boundaries share the same modal displacement.

In principle, Eqn. (16) should be solved for any wave vector k. Two special cases arise when analyzing the geometrical features of the optimal layouts shown in the numerical section. They are (i) square symmetry (square cell, symmetric with respect to horizontal, vertical and diagonal lines) and (ii) anti-symmetry of a "zigzag" cell. According to [52], the former can be characterized restricting the analysis to wave vectors $\mathbf{k}$ lying along the paths $\Gamma-X-M-\Gamma$ of the so-called first Brillouin zone, see [16], whereas $\Gamma-X / Y-M-\Gamma$ applies to the latter, see Figure 1.

The first 6 eigenvalues will be solved for the selected wave vectors to generate the so-called band diagram. For each eigenvalue, the $\omega^{2}-\mathbf{k}$ locus defines the relevant dispersion curve of the Floquet-Bloch spectrum. The frequency range covered by a single dispersion curve is denoted as frequency band. Two dispersion curves can cross each other or can be separated by a frequency range referred to as full band gap. A partial or directional band gap refers to a frequency separation that is restricted to a certain $\mathbf{k}$-direction. For a homogenous material, eigenmodes are expected for any frequency. A micro-structured material with full phononic band gap exhibits a range of frequency with no corresponding eigenmode, meaning that no in-plane mode will propagate for these frequencies. In case of partial band gap, this property is limited to a certain direction in the plane of the cell. 


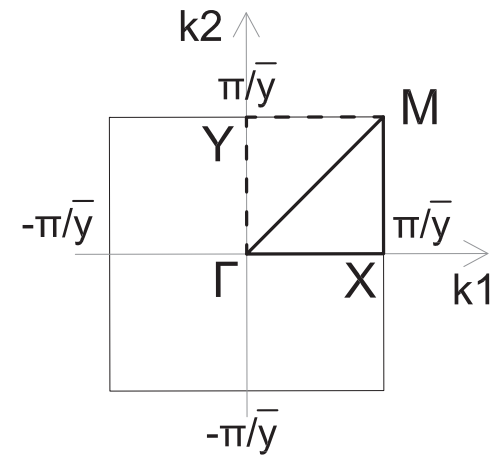

Figure 1: Reciprocal diagram: Brillouin zone and reduced regions in case of square symmetric unit cells (with borders $\Gamma-X-M-\Gamma)$ and in case of anti-symmetric "zigzag" cells $(\Gamma-X / Y-M-\Gamma)$.

\section{Numerical simulations}

Numerical investigations are performed on the square unit cell with $\bar{y}=10 \mathrm{~mm}$ represented in Figure 2, adopting a mesh with 16000 elements. A circle with radius $\bar{y} / 6 \leq r \leq \bar{y} / 3$ splits the design domain into two regions: the inner part is made of material with volume fraction $x_{m i n} \leq x_{i n i, 1} \leq V_{f}$, whereas the outer consists of material $x_{\min } \leq x_{i n i, 2} \leq V_{f}$. Local optima are generated adopting $\beta=10^{6}$ in the objective function of Eqn. (12) and initializing the optimization from different sets of $r, x_{i n i, 1}, x_{i n i, 2}$, available amount of material $V_{f}$ and hard material $V_{h}$. A reference material with Young's modulus $E=10000 \mathrm{~N} / \mathrm{mm}^{2}$, Poisson's ratio $\nu=0.2$ and volumetric mass density $\rho=1000 \mathrm{~kg} / \mathrm{m}^{3}$ is considered when dealing with the distribution of material and void, see Sections 4.1-4.3. Optimal layouts are presented through maps of the distribution of the minimization variables: black stands for full material, whereas white stands for "void", i.e. regions of minimum density with $x_{\min }=10^{-3}$. The achieved solutions are endowed with band diagrams plotted in terms of the non-dimensional frequency of the incident waves, i.e. $\omega / \omega_{0}$ with $\omega_{0}=v_{p} / \bar{y}$ and $v_{p}=\sqrt{\frac{\lambda+2 \mu}{\rho}}$. A full band gap is highlighted in the diagrams as the maximum frequency interval detected between the dispersion curves, i.e. for all the wave vectors $\mathbf{k}$ of the considered paths $\Gamma-X$ - 


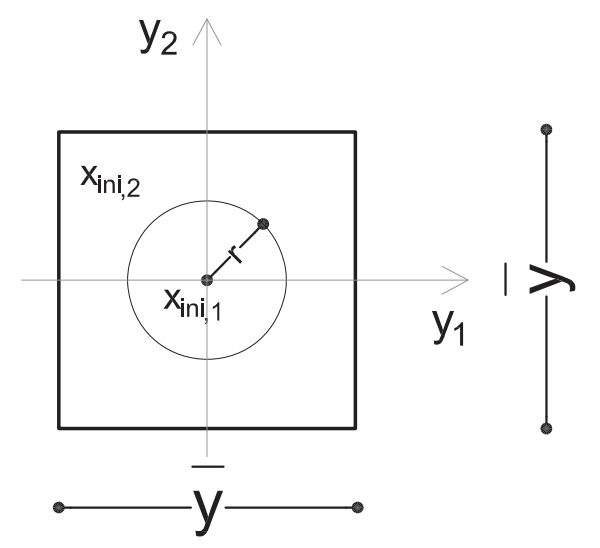

Figure 2: Initial guess. Mesh of 16000 elements

$M-\Gamma$ or $\Gamma-X / Y-M-\Gamma$. Directional band gaps are highlighted as the maximum frequency intervals detected for the wave vectors $\mathbf{k}$ lying on a single segment or two consecutive segments of the above paths.

In Section 4.4 the distribution of two materials and void is addressed. Two combinations are considered. In combination 1 , the hard material is the same defined above i.e. $E_{h 1}=E, \nu_{h 1}=\nu$, $\rho_{h 1}=\rho$, whereas for the soft material $E_{s 1}=E_{h 1} / 10, \nu_{s 1}=\nu_{h 1}, \rho_{s 1}=\rho_{h 1} / 10$. In combination 2, the hard material reads $E_{h 2}=110000 \mathrm{~N} / \mathrm{mm}^{2}, \nu_{h 2}=0.2, \rho_{h 2}=8960 \mathrm{~kg} / \mathrm{m}^{3}$ and the soft one $E_{s 2}=1800 \mathrm{~N} / \mathrm{mm}^{2}, \nu_{s 2}=\nu_{h 2}, \rho_{s 2}=1290 \mathrm{~kg} / \mathrm{m}^{3}$. The latter set of values stands for copper (hard material) and plastic resin (soft material), as inspired by the 3D-printing application in [53]. In both cases optimal layouts are presented through material density maps where black means hard phase, grey is the soft phase and white is "void". The non-dimensional frequency is computed with respect to the speed of a $P$-wave in an homogenous medium made of the relevant hard material phase.

For each optimal auxetic micro-structure made of material and void, the components of the 


\begin{tabular}{l|cccccc} 
& $E_{11}^{H}$ & $E_{22}^{H}$ & $E_{33}^{H}$ & $E_{12}^{H}$ & $E_{13}^{H}$ & $E_{23}^{H}$ \\
\hline Fig. 3 & 632 & 632 & 41 & -205 & 0 & 0 \\
Fig. 5 & 514 & 514 & 384 & -254 & 0 & 0 \\
\hline Fig. 7 & 637 & 595 & 17 & -476 & 16 & -13 \\
Fig. 9 & 1392 & 1390 & 247 & -987 & 6 & -5 \\
\hline Fig. 10 & 269 & 270 & 5 & -206 & -62 & 62 \\
Fig. 12 & 584 & 585 & 31 & -414 & 229 & -229 \\
Fig. 15 & 1179 & 1184 & 131 & -782 & 642 & -644 \\
Fig. 18 & 1268 & 1268 & 197 & -917 & -302 & 301
\end{tabular}

Table 1: Components of the homogenized elasticity tensor $\mathbf{E}^{H}$ for each one of the micro-structures achieved by distribution of material and void (values in $\mathrm{MPa}$ ).

\begin{tabular}{l|cccccc} 
& $E_{11}^{H}$ & $E_{22}^{H}$ & $E_{33}^{H}$ & $E_{12}^{H}$ & $E_{13}^{H}$ & $E_{23}^{H}$ \\
\hline Fig. 19 & 1321 & 1321 & 522 & -910 & 129 & -129 \\
Fig. 20 & 642 & 643 & 53 & -488 & 323 & -323 \\
Fig. 21 & 523 & 523 & 50 & -388 & 61 & -61 \\
Fig. 23 & 4733 & 4700 & 220 & -3526 & -1166 & 1162 \\
Fig. 24 & 7253 & 7175 & 275 & -5637 & 66 & -70
\end{tabular}

Table 2: Components of the homogenized elasticity tensor $\mathbf{E}^{H}$ for each one of the micro-structures achieved by distribution of two materials and void (values in $\mathrm{MPa}$ ).

homogenized elasticity tensor $\mathbf{E}^{H}$ are reported in Table 1. Table 2 refers to optimal results achieved by distribution of two materials and void.

An overview of the optimal micro-structures achieved by the proposed numerical tool is given in Table 3 and Table 4 for single material and bi-material design, respectively. In each row, the topology of each one of the unit cells retrieved by the implemented numerical tool is reported, along with the effective Poisson's ratio(s), the classification according to the geometry and the type of band gap detected through the eigenvalue analysis. When a partial band gap arises along two consecutive segments of the considered path, these are specified in the table. 
No remarkable change is expected in terms of optimal shapes when using different materials in the single-phase design, as classically found in structural topology optimization at least for reasonable values of the Poisson's ratio, see e.g. [27]. Conversely, the presence of a hard and a soft phase strongly affects the achieved layouts. The present study is limited to a few combinations of materials to point out some general features of the multi-phase optimization and of the band gap properties of the optimal shapes. Of course, the proposed numerical procedure can be used to explore a wider set of solutions using different constituent phases.

\subsection{Re-entrant micro-structures}

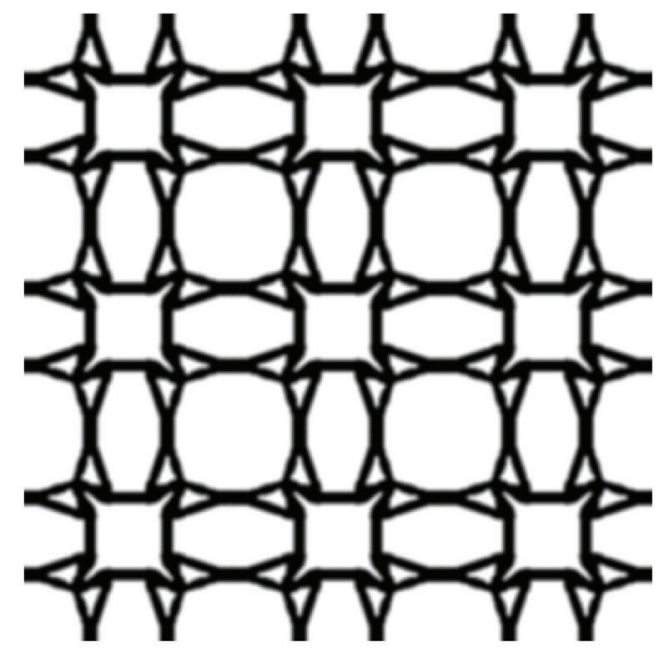

(a)

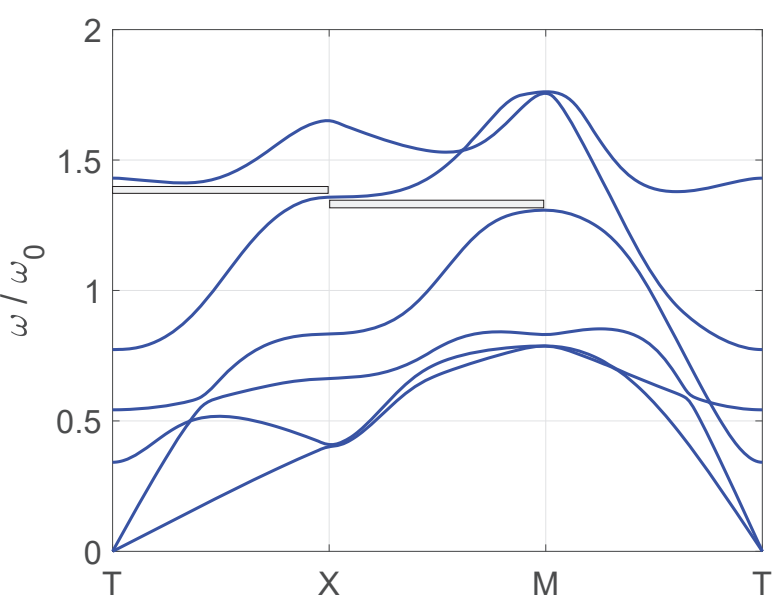

(b)

Figure 3: Re-entrant micro-structure with $\nu *=-0.33\left(V_{f}=0.3\right): 3 \times 3$ array of cells (a) and band diagram (b).

Two optimal micro-structures are shown whose auxetic behavior depends on the geometry exhibiting re-entrant members. Both cells are square symmetric, with equal effective Poisson's ratios $\nu_{12}^{*}=\nu_{21}^{*}=\nu *$, as aimed by the implemented objective function. Figure 3(a) shows a $3 \mathrm{x}$ 3 array of cells of an optimal micro-structured material with $\nu *=-0.33$ that has been achieved 

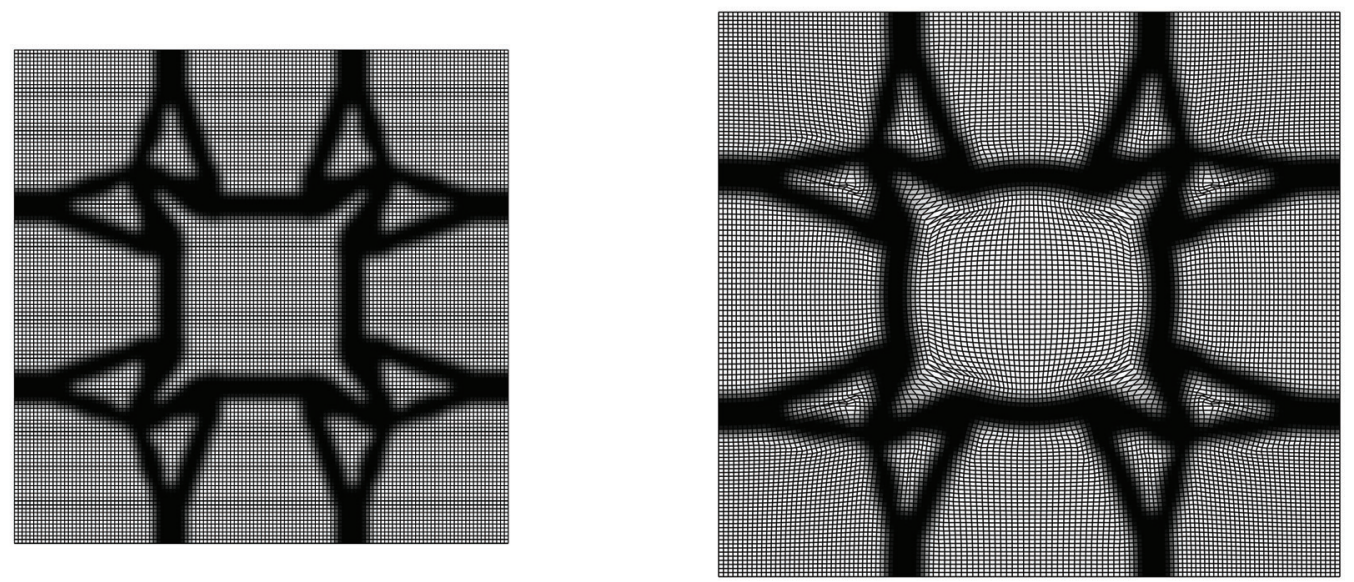

(a)

(b)

Figure 4: Re-entrant micro-structure with $\nu *=-0.33$ : underformed shape (a) vs. magnified displacement plot of the unit cell undergoing $\varepsilon_{11}$.

enforcing $V_{f}=0.3$. Figure 4 shows the magnified displacement plot of the unit cell subjected to a positive $\varepsilon_{11}$ : the cell undergoes a stretching in the orthogonal direction that is one third of the applied one. The applied strain opens the $\mathrm{V}$-shaped corners in the central ring, thus providing the expected auxetic behavior. The band diagram of this layout is reported in Figure 3(b): a minor directional band gap arises between the 5-th and the 6-th eigenmode for wave vectors of the type $\mathbf{k}=\left(k_{1}, 0\right)$ and between the 4 -th and the 5 -th eigenmode for wave vectors of the type $\mathbf{k}=\left(\pi / \bar{y}, k_{2}\right)$, see the coloured areas in the regions $\Gamma-X$ and $X-M$ of the diagram, respectively.

Figure 5(a) shows an alternative $3 \times 3$ array of cells of an optimal micro-structured material with $\nu *=-0.49$ that has been achieved for the same volume fraction used above, but a different initialization of the minimization algorithm. Figure 6 shows the magnified displacement plot of the unit cell subjected to a positive strain along the horizontal direction: the cell undergoes a 


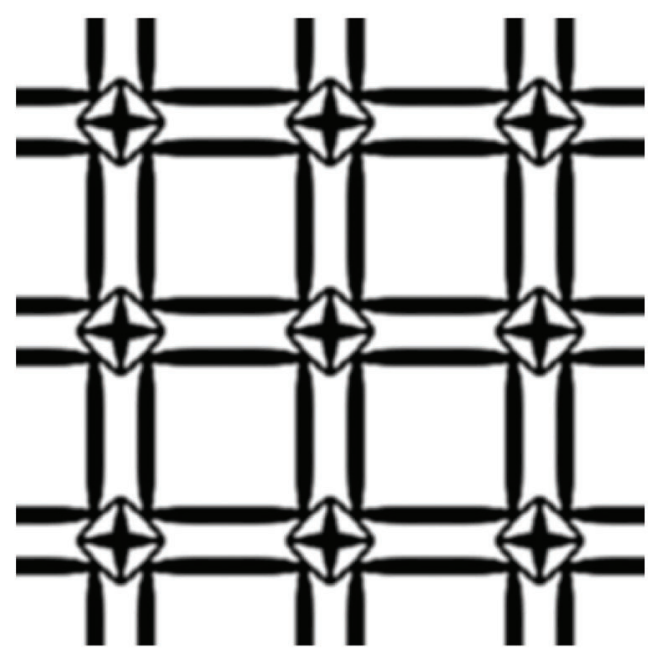

(a)

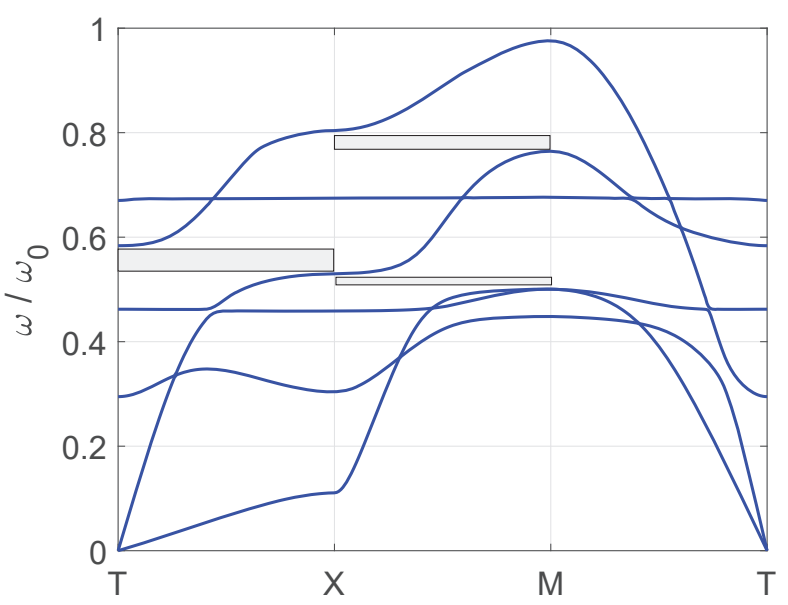

(b)

Figure 5: Re-entrant micro-structure with $\nu *=-0.49\left(V_{f}=0.3\right): 3 \times 3$ array of cells (a) and band diagram (b).

stretching in the vertical direction that is almost half of the applied one. The auxetic behavior is provided by the flexure of the four sides of the thin rhombic core of the micro-structure. Again, minor directional band gaps can be outlined in the band diagram, see Figure 5(b). Waves with vectors of the type $\mathbf{k}=\left(k_{1}, 0\right)$ can not propagate in the range between the 4 -th and the 5 -th eigenfrequency, whereas waves with vectors of the type $\mathbf{k}=\left(\pi / \bar{y}, k_{2}\right)$ are stopped between the 5 -th and the 6 -th eigenfrequency.

According to [9], the achieved optimal layouts could be classified as re-entrant models. Both micro-structures feature re-entrant cellular configurations with flexure of the diagonal ribs, see e.g. the auxetic honeycombs in [14]. A tuning of the thickness of the elements could be implemented on the outlined topologies to govern their auxetic behavior, acting on the filter radius for the density field $r_{f}$.

It must be remarked that optimal star-shaped micro-structures achieved through topology optimization can be affected by the arising of undesired "hinges", i.e. weak regions that connect 


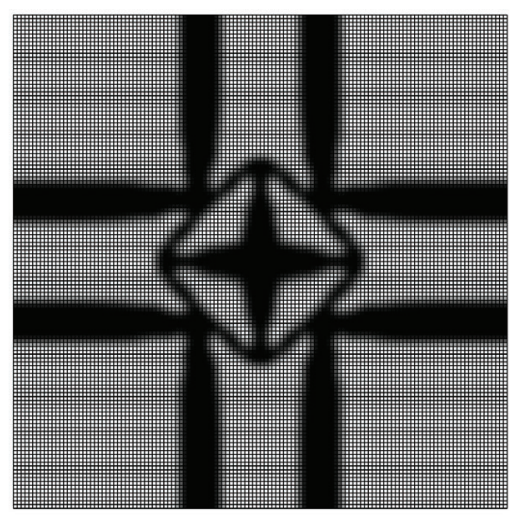

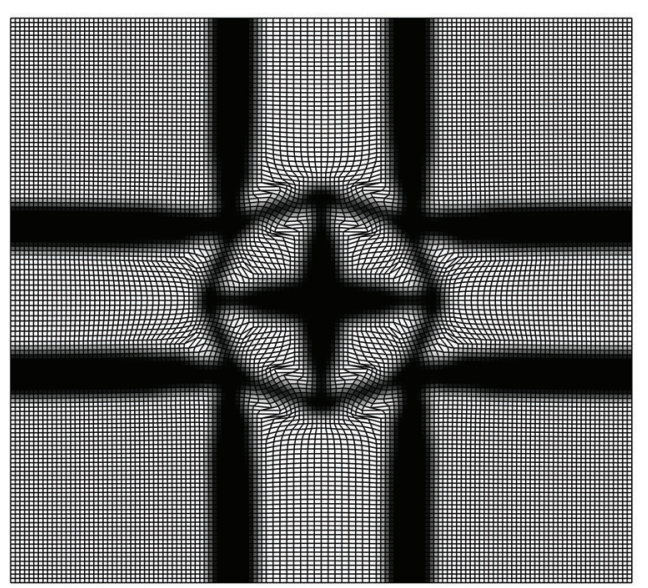

(b)

Figure 6: Re-entrant micro-structure with $\nu *=-0.49$ : underformed shape (a) vs. magnified displacement plot of the unit cell undergoing $\varepsilon_{11}(\mathrm{~b})$.

bars to allow for the auxetic deformation through a lumped mechanism, see e.g. [34]. Hinges are an issue both for manufacturing and strength. If the density filter fails in preventing such kind of numerical instabilities, alternative approaches may be implemented in conjunction with the considered objective function, see Section 2.1.

\subsection{Anti-chiral tessellations of basic units with rotational symmetry}

In this section, optimal auxetic micro-structures are shown that consist of anti-chiral tessellations of tetrachiral units, i.e. basic geometries with rotational symmetry of order 4 .

Figure 7(a) shows an optimal anisotropic micro-structure achieved for the same volume fraction used in the previous section, i.e. $V_{f}=0.3$. The auxetic deformation is not of the same amplitude when stretching the material along the horizontal direction or the vertical one. The minimum effective Poisson's ratio is $\nu *_{12}=-0.80$, whereas the maximum reads $\nu *_{21}=-0.75$. Focusing on 


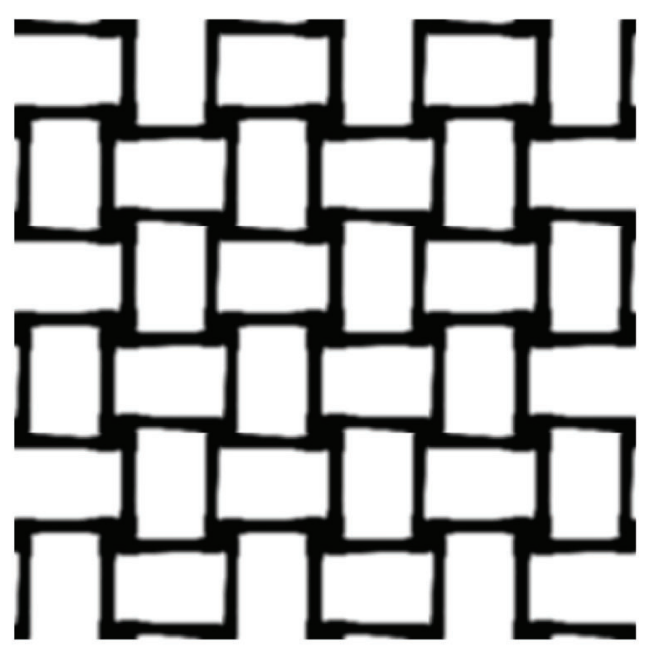

(a)
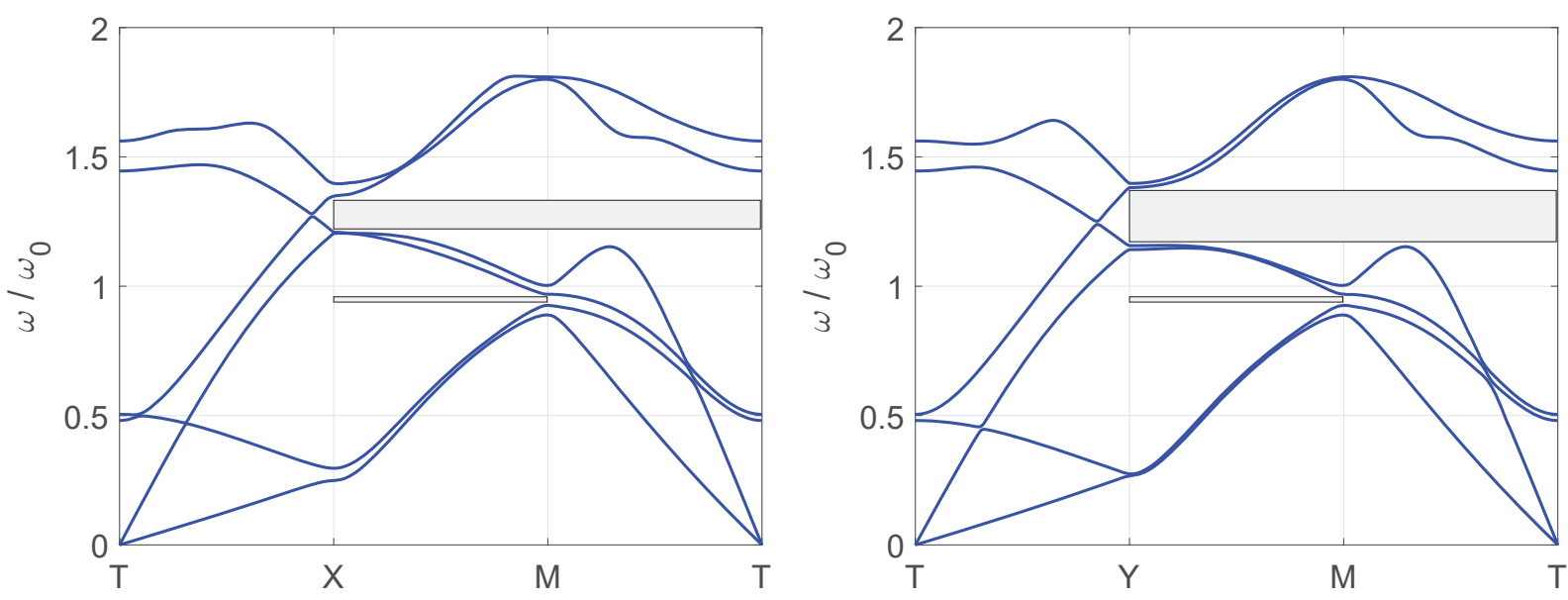

(b)

Figure 7: Anti-chiral auxetic micro-structure with $\nu *_{12}=-0.80, \nu *_{21}=-0.75\left(V_{f}=0.3\right): 3 \times 3$ array of cells (a) and band diagrams (b-c).

$\nu *_{12}$, Figure 8 shows a magnified deformed shape of the unit cell subjected to a positive strain along the horizontal direction: the tetrachiral unit, i.e. the inner node and its four eccentric arms, undergoes a rotational deformation that provides a remarkable vertical elongation. The 


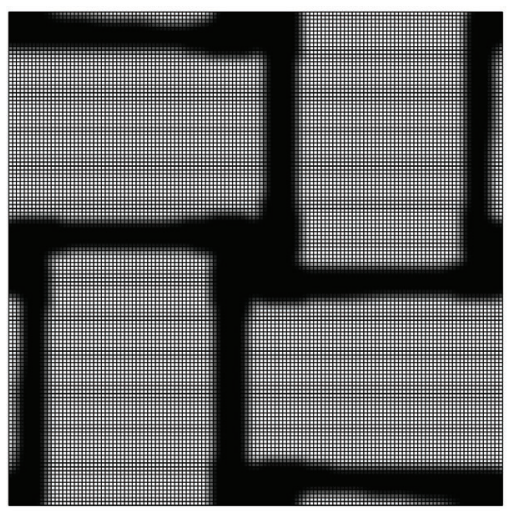

(a)

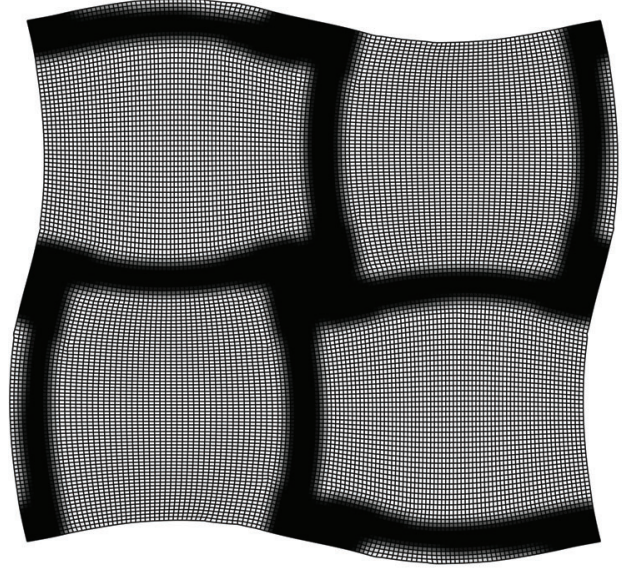

(b)

Figure 8: Anti-chiral auxetic micro-structure with $\nu *_{12}=-0.80, \nu *_{21}=-0.75$ : underformed shape (a) vs. magnified displacement plot of the unit cell undergoing $\varepsilon_{11}$.

achieved micro-structure is quite similar to the equivalent two-dimensional composite sketched in [54] when investigating the three-dimensional design of piezocomposites with optimal performance for hydrophone applications. Reference is also made to the anti-chiral honeycomb structures investigated in [55] and to the missing rib foam model firstly proposed in [56]. Figure 7(b-c) shows the relevant band diagrams. A directional band gap arises between the 4 -th and the 5 -th eigenmode for wave vectors in $X / Y-M-\Gamma$.

Figure 9(a) shows an optimal micro-structure achieved for twice the volume fraction used for the previous simulation, now $V_{f}=0.6$. The topology looks like the previous one, but it features mechanical properties that are slightly different. This stiffer micro-structure is nearly orthotropic. While a normal strain produces also a (minor) shear stress when applied to the micro-structure reported in Figure 7(a), no remarkable coupling is found for the square symmetric material in 


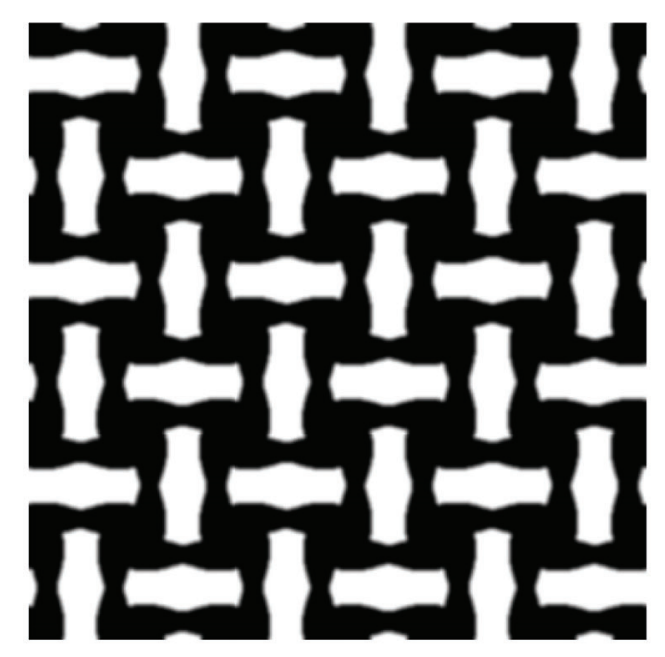

(a)
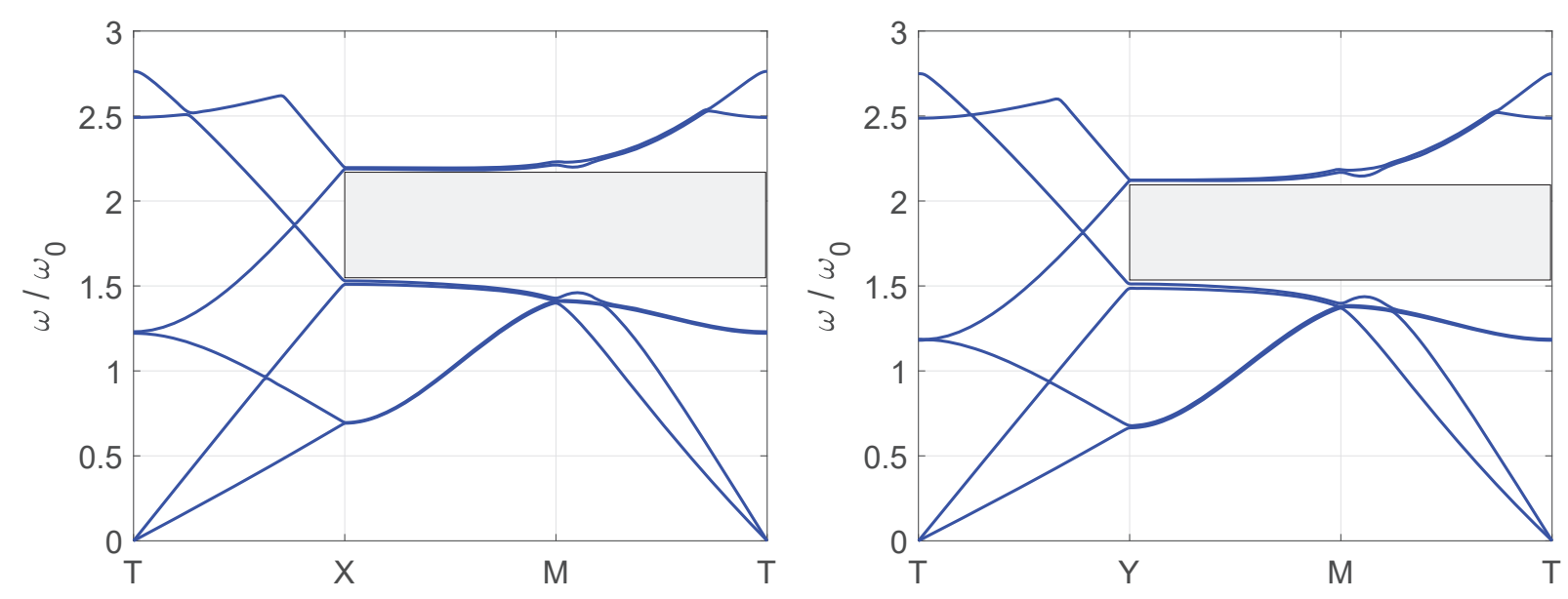

(b)

(c)

Figure 9: Anti-chiral auxetic micro-structure with $\nu *=-0.71\left(V_{f}=0.6\right): 3 \times 3$ array of cells (a) and band diagrams (b-c).

Figure 9(a), see Table 1. The increased volume fraction is responsible for a smaller Poisson effect. The effective Poisson's ratio reads $\nu_{12}^{*}=\nu_{21}^{*}=\nu *=-0.71$. Referring to the band diagrams shown in Figure 9(b-c), a directional band gap arises between the 4-th and the 5-th eigenmode for wave 
vectors in $X / Y-M-\Gamma$, as already found in Figure $7(\mathrm{~b}-\mathrm{c})$. The increased amount of material affects both stiffness and mass of the optimal micro-structure, resulting in an increased extension of the directional band gap that also has a lower central frequency. This suggests the adoption of the volume fraction $V_{f}$ as a heuristic parameter to customize both features of the observed directional stop band.

\subsection{Chiral tessellations of basic units with rotational symmetry}

Several optimal layouts found through the implemented approach consist of chiral tessellations of tetrachiral basic units. Figure 10(a) shows an example that has been synthesized enforcing $V_{f}=0.15$.

The equivalent Poisson's ratio is $\nu_{12}^{*}=\nu_{21}^{*}=\nu *=-0.76$. A non negligible coupling arises between normal strains and shear stresses in the achieved anisotropic micro-structure, see values of $E_{13}^{H}, E_{23}^{H}$ in Table 1. Figure 11 shows the magnified deformed shape of the unit cell subjected to a positive $\varepsilon_{11}$ : the micro-structure undergoes a stretching in the orthogonal direction because of the rotation of the tetrachiral cell, which consists of an inner core and four eccentric arms. Optimal tessellations of tetrachiral units have been found in [57] when designing optimal auxetic structures accounting for geometrical nonlinearities. Indeed, chiral honeycombs have been numerically and experimentally demonstrated to exhibit a strong auxetic behaviour not only under small deformation but also under finite deformation. Figure 10(b-c) reports the band diagrams of the achieved micro-structure: three minor directional band gaps arise for different frequencies and wave vectors that are all in $X-M$.

Figure 12(a) shows a very similar topology that has been synthesized for $V_{f}=0.3$. With respect to the previous micro-structure, the equivalent Poisson's ratio is slightly bigger $\left(\nu_{12}^{*}=\nu_{21}^{*}=\nu *=\right.$ -0.71 ) and the coupling between normal strains and shear stresses due to the anisotropy of the cell increases, see Table 1. Figure 12(b-c) shows that a stop band arises between the 5 -th and the 6-th eigenmode. Within this band gap, a strong attenuation is expected independently of the direction 


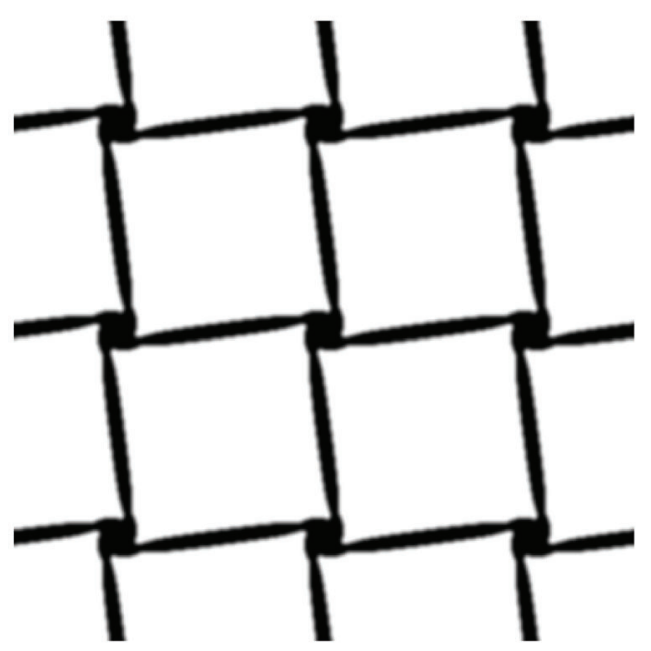

(a)
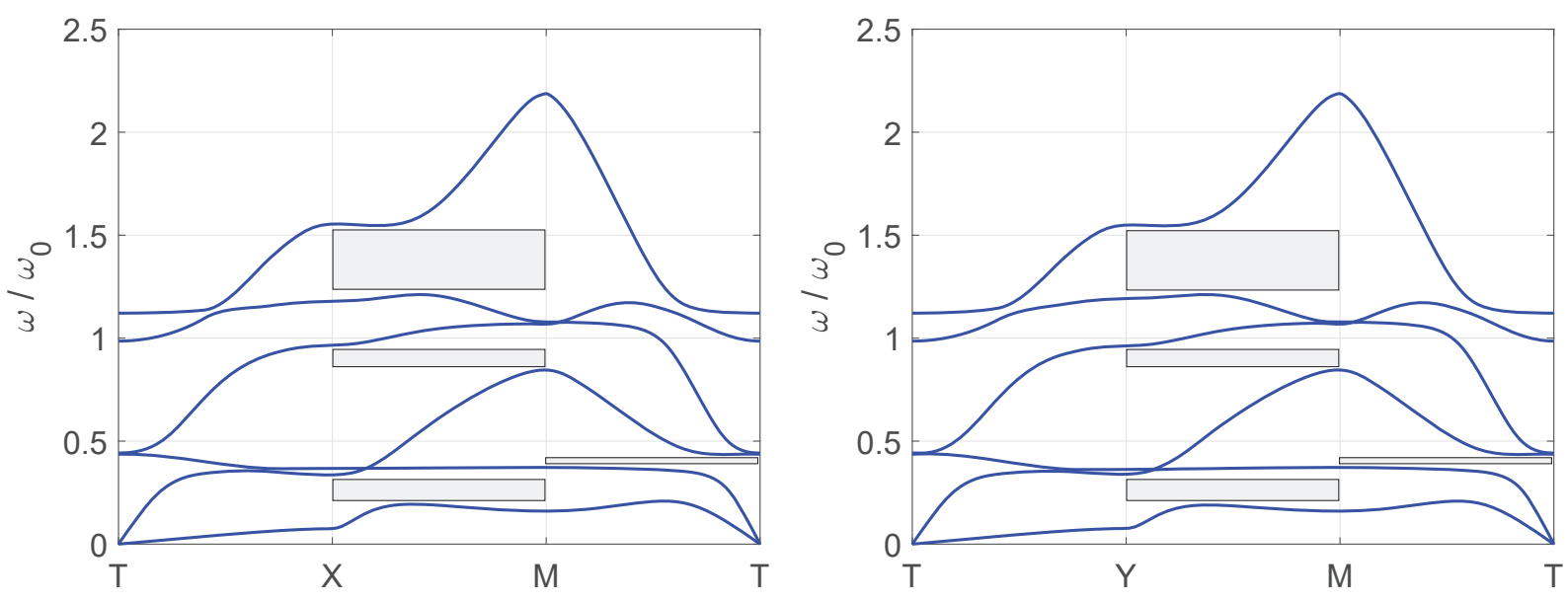

(b)

(c)

Figure 10: Chiral auxetic micro-structure with $\nu *=-0.76\left(V_{f}=0.15\right): 3 \times 3$ array of cells (a) and band diagrams (b-c).

of the wave vector. Figure 13 shows eigenmodes from the 3 rd to the 6 th at $k_{x}=k_{y}=0(\Gamma)$. The first and second modes correspond to rigid-body modes. The 3rd and the 4th eigenmode show that wave propagation for these polarizations occurs mostly through bending of the eccentric arms. 


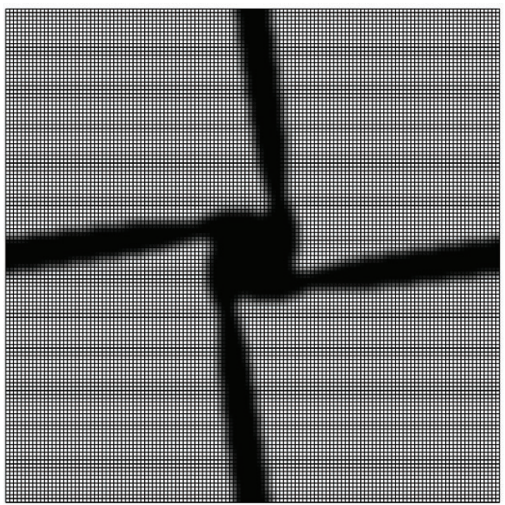

(a)

Figure 11: Chiral auxetic micro-structure with $\nu *=-0.76$ : underformed shape (a) vs. magnified displacement plot of the unit cell undergoing $\varepsilon_{11}$.

The 5th mode is characterized by a rotation of the chiral basic unit around its center, whereas the 6th one involves again bending of the ligaments. Figure 14 shows the first five eigenmodes at $k_{x}=\pi / \bar{y}, k_{y}=0(X)$. At this location, the fifth mode is the first for which relevant deformations of the inner core can be observed. Hence, the mode corresponding to the lower bound of the stop band is rotational (at $\Gamma$ ) or related to relevant deformations of the core within the unit cell (at $X)$. This is in agreement with what found by the work by [58] with respect to phononic properties of hexagonal chiral lattices. It also points out that the core of chiral lattices may behave as an internal inclusion, whose stiffness and mass strongly affects band gap characteristics.

Figure 15(a) shows a chiral tessellation that has been synthesized for $V_{f}=0.6$, see also [39]. The homogenized constitutive tensor denotes a remarkable coupling between normal strains and shear stresses, see Table 1. The equivalent Poisson's ratio is $\nu_{12}^{*}=\nu_{21}^{*}=\nu *=-0.66$. Figure 


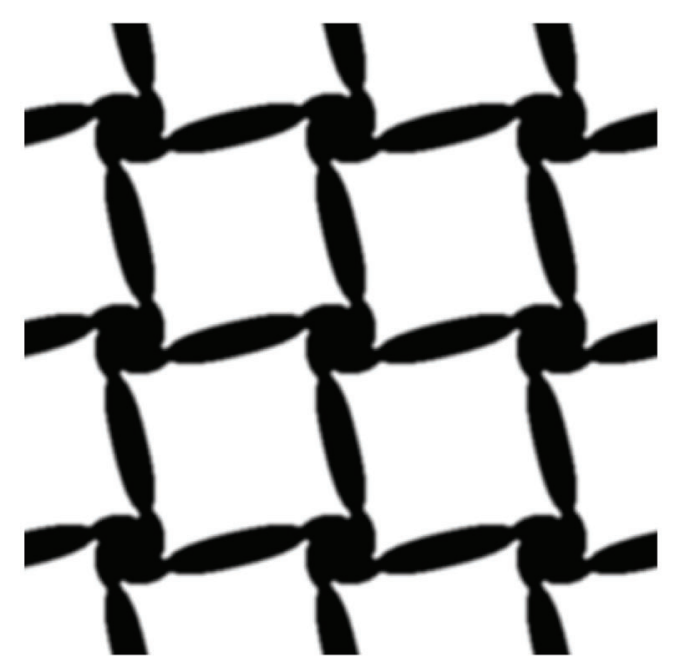

(a)
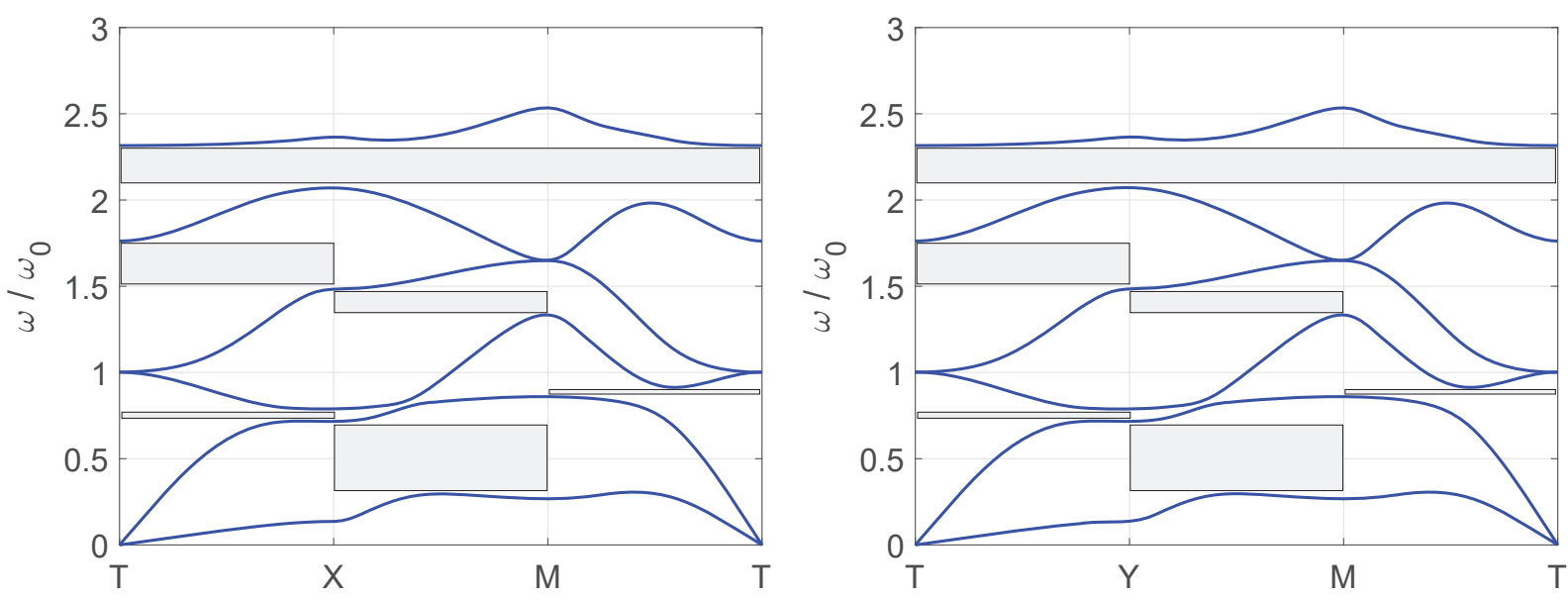

(b)

(c)

Figure 12: Chiral auxetic micro-structure with $\nu *=-0.71\left(V_{f}=0.3\right): 3 \times 3$ array of cells (a) and band diagrams (b-c).

16 shows the magnified deformed shape of the tetrachiral unit cell subjected to a positive strain along the horizontal direction: a rotational deformation arises to provide the expected vertical stretching. A stop band arises between the 3-rd and the 4-th eigenmode, see Figure 15(b-c). 

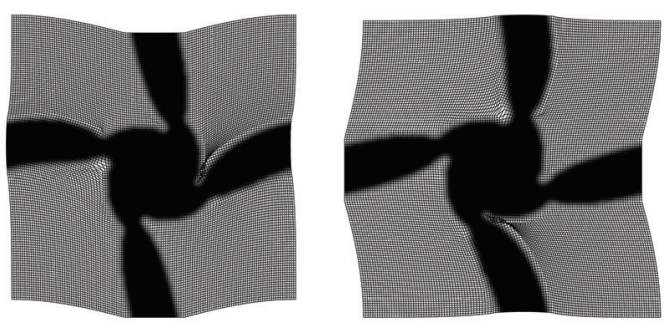

(a)

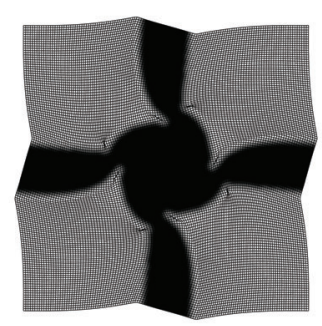

(b)

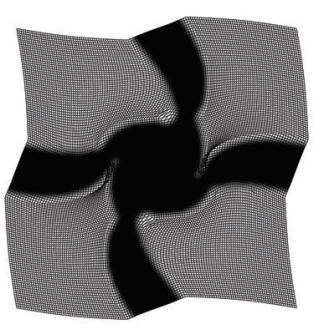

(c) (d)

Figure 13: Chiral auxetic micro-structure with $\nu *=-0.71\left(V_{f}=0.3\right)$ : 3rd (a), 4th (b), 5th (c), 6th (d) eigenmode for $k_{x}=k_{y}=0(\Gamma)$.
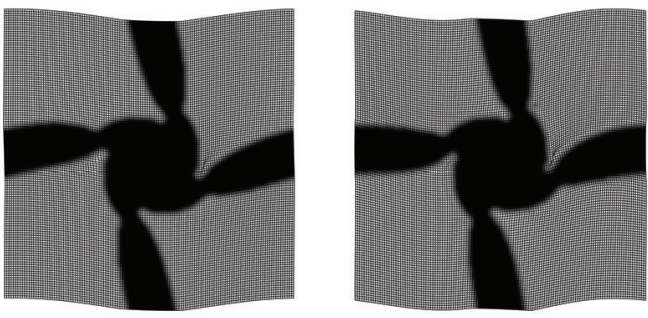

(a)

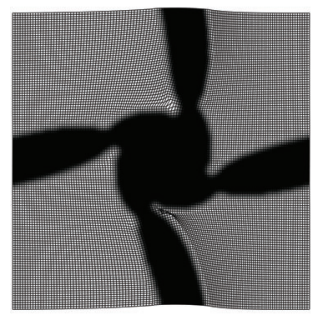

(b)

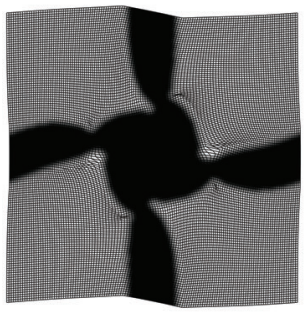

(c)

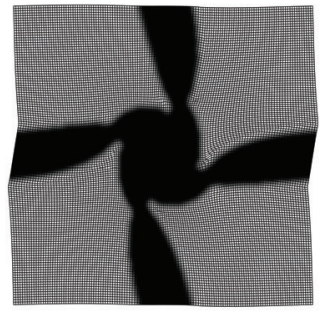

(d) (e)

Figure 14: Chiral auxetic micro-structure with $\nu *=-0.71\left(V_{f}=0.3\right)$ : 1st (a), 2nd (b), 3rd (c), 4th (d), 5th (e) eigenmode for $k_{x}=\pi / \bar{y}, k_{y}=0(X)$.

Again, the mode corresponding to the lower bound of the stop band is rotational (at $\Gamma$ ), see the 3-rd eigenmode of the unit cell represented in Figure 17.

For the same volume fraction, another chiral tessellation of tetrachiral basic units can be alternatively retrieved, see Figure 18. The auxetic behavior is improved with respect to the previous micro-structure $\left(\nu_{12}^{*}=\nu_{21}^{*}=\nu *=-0.72\right)$, but only two minor directional band gaps arise for wave vectors of the type $\mathbf{k}=\left(k_{1}, 0\right)$. 


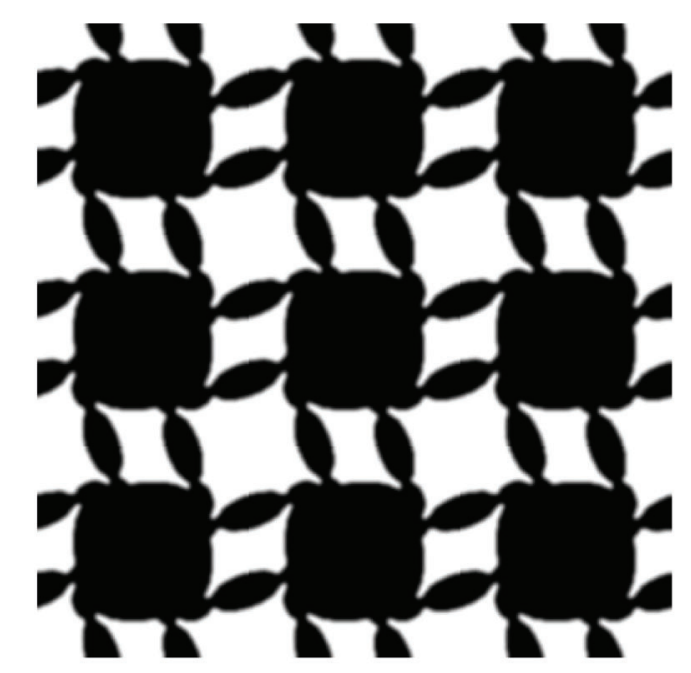

(a)
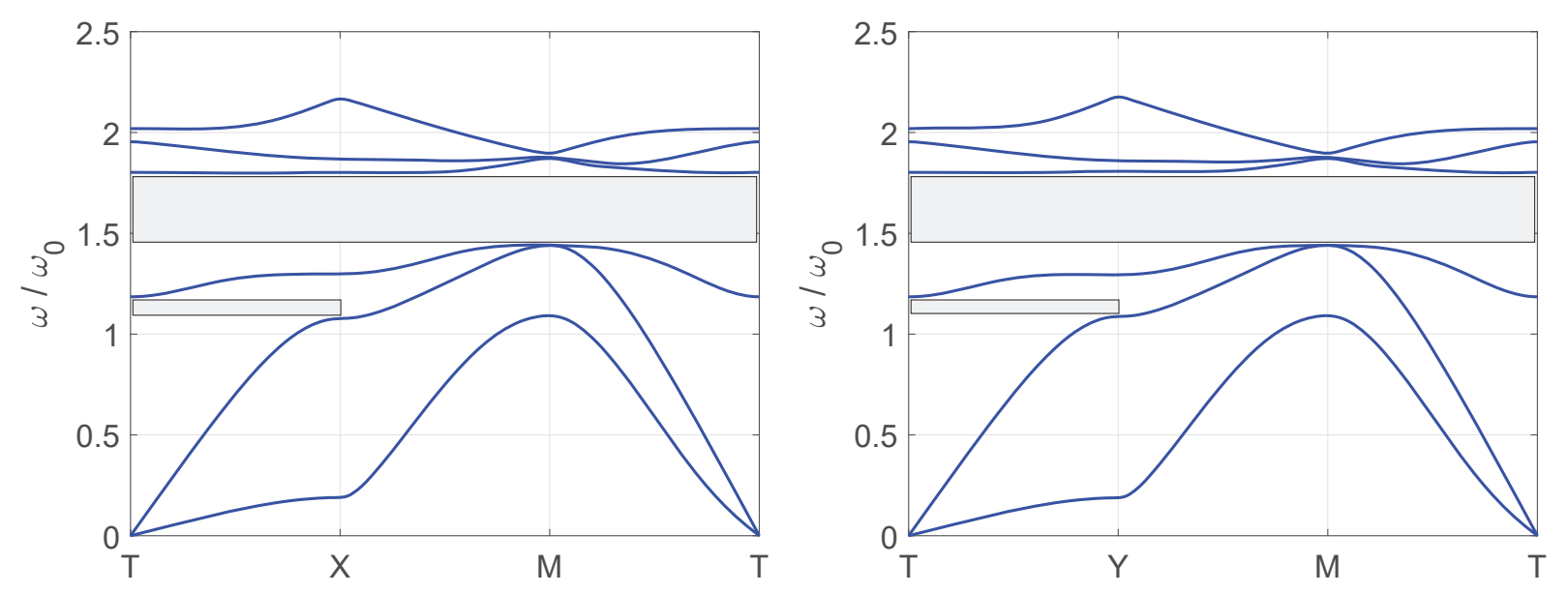

(b)

(c)

Figure 15: Chiral auxetic micro-structure with $\nu *=-0.66\left(V_{f}=0.6\right): 3 \times 3$ array of cells (a) and band diagrams (b-c).

\subsection{Variations using bi-material design}

An additional set of investigations has been performed considering the optimal distribution of two materials and void. At first the combination 1 of the hard and soft material components is 

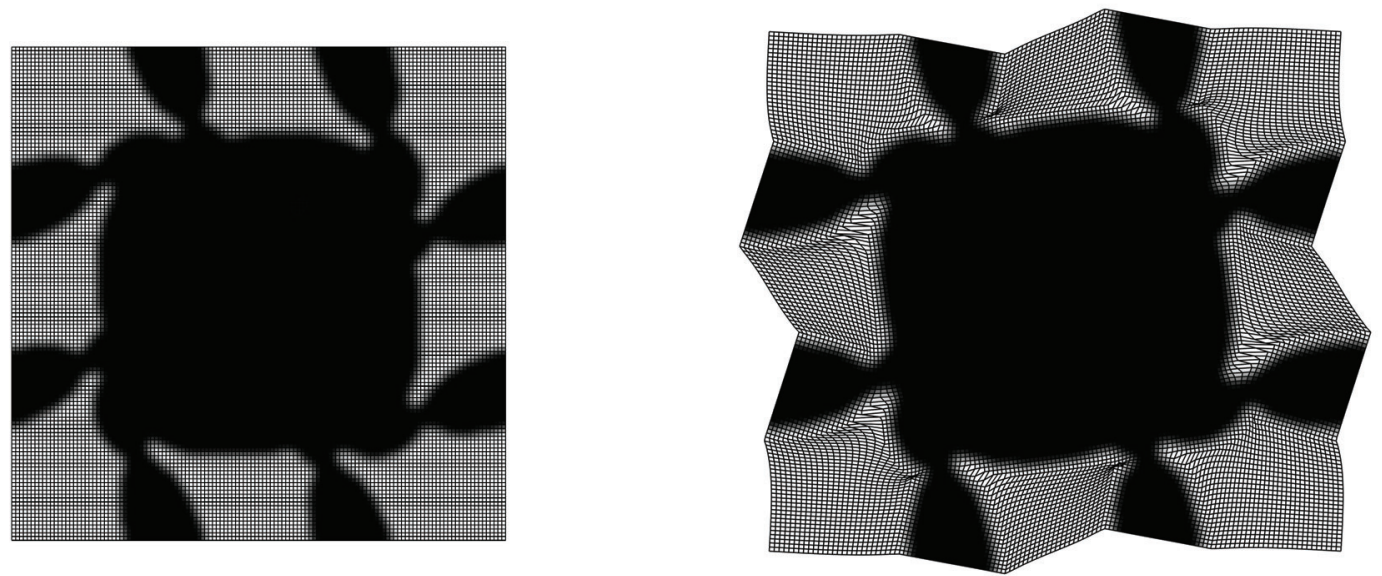

(a)

(b)

Figure 16: Chiral auxetic micro-structure with $\nu *=-0.66$ : underformed shape (a) vs. magnified displacement plot of the unit cell undergoing $\varepsilon_{11}(\mathrm{~b})$.
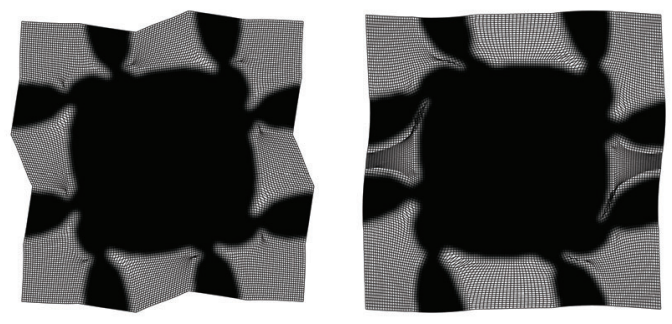

(a)

(b)

Figure 17: Chiral auxetic micro-structure with $\nu *=-0.66$ : 3rd (a) and 4th (b) eigenmode for $k_{x}=k_{y}=0(\Gamma)$.

addressed. Figure 19(a) shows a bi-material auxetic micro-structure with $\nu_{12}^{*}=\nu_{21}^{*}=\nu *=-0.69$ that has been achieved enforcing a maximum amount of material $V_{f}=V_{h 1}+V_{s 1}=0.8$ with maximum volume of hard material $V_{h 1}=0.6$. The optimal design is a variation of the tessellation 


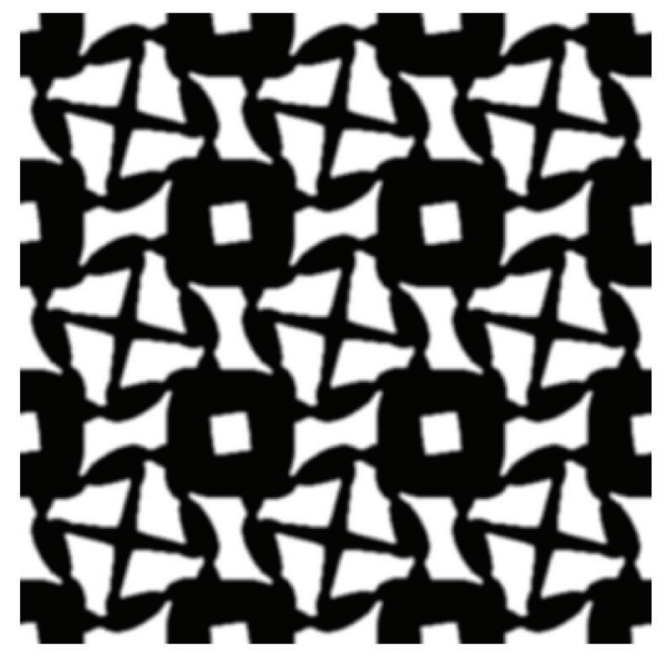

(a)
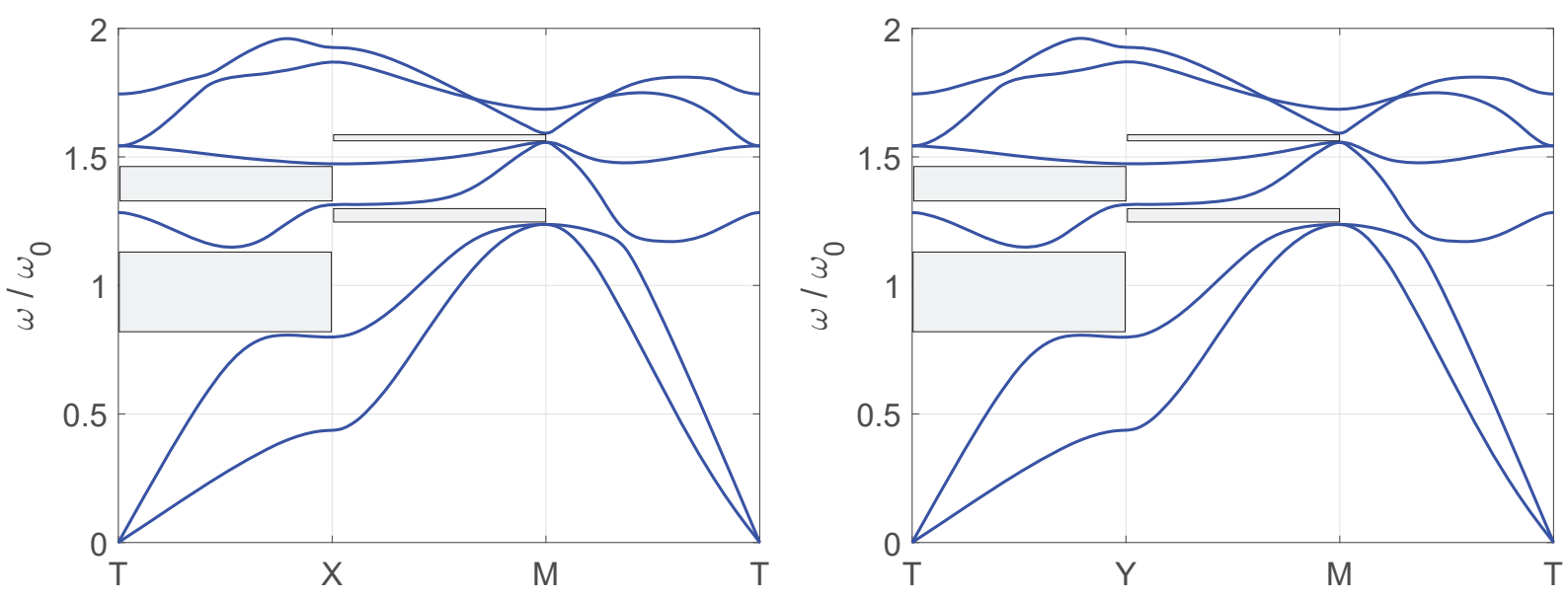

(b)

(c)

Figure 18: Chiral auxetic micro-structure with $\nu *=-0.72\left(V_{f}=0.6\right): 3 \times 3$ array of cells (a) and band diagrams (b-c).

of tetrachiral basic units of Figure 18 that has been achieved for the distribution of hard material only and void. In this bi-material anisotropic structure, the hard material (black regions in the density plot) acts as a rigid coating for the soft material (grey zones). The relevant band diagram 


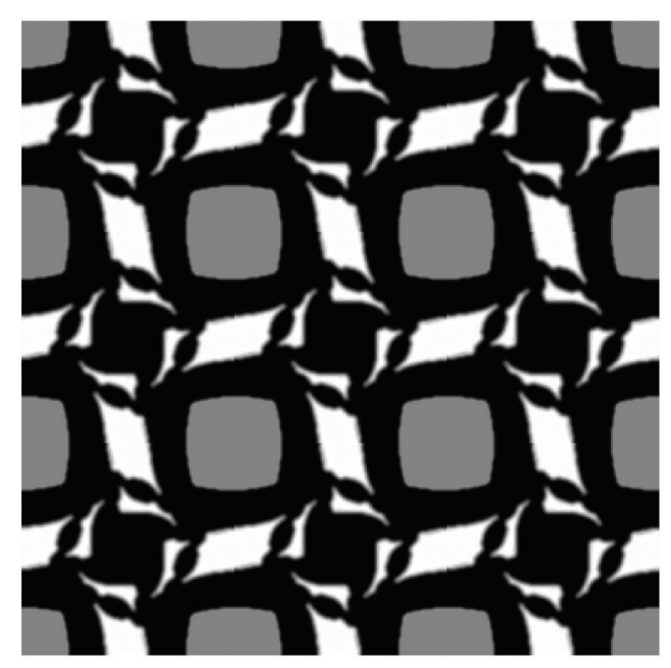

(a)
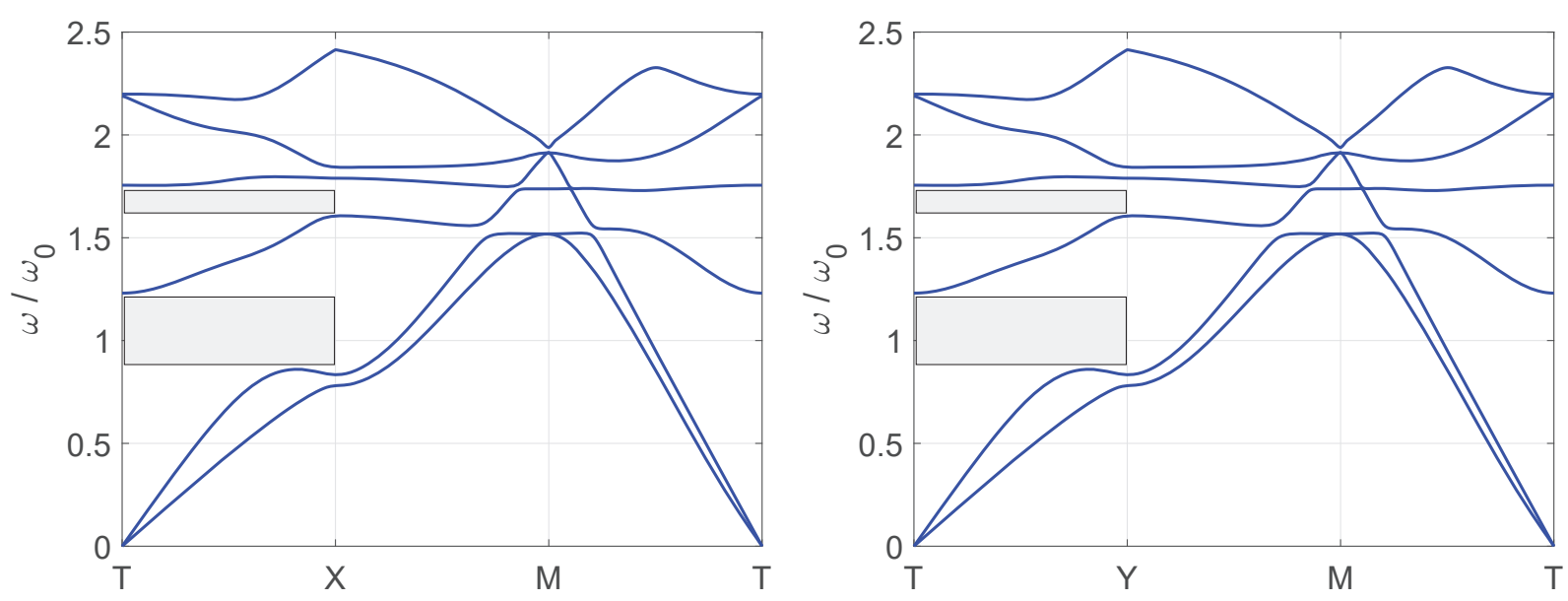

(b)

(c)

Figure 19: Bi-material auxetic micro-structure with $\nu *=-0.69\left(V_{f}=0.8, V_{h 1}=0.6\right): 3 \times 3$ array of cells (a) and band diagrams (b-c).

is quite similar to the one found for the previous micro-structure, see Figure 19(b-c) as compared to Figure 18(b-c).

The hard material mainly plays as a coating for the soft one also in the optimal topology 


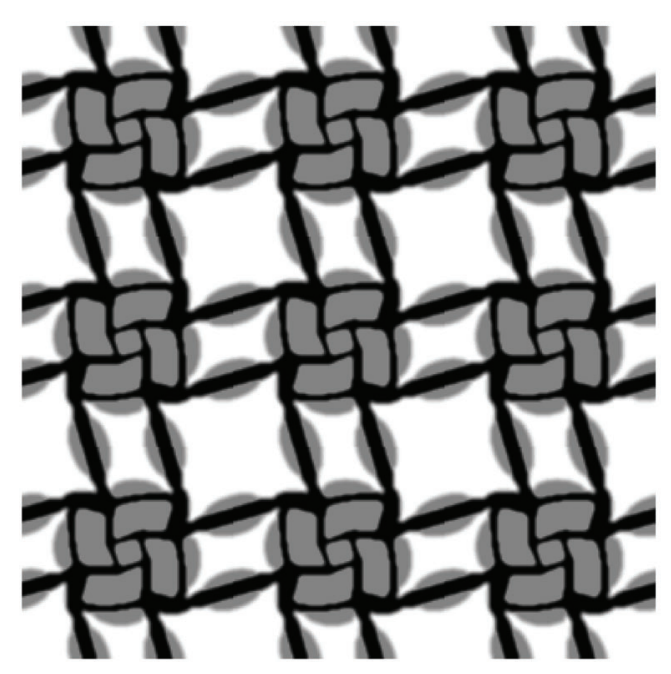

(a)
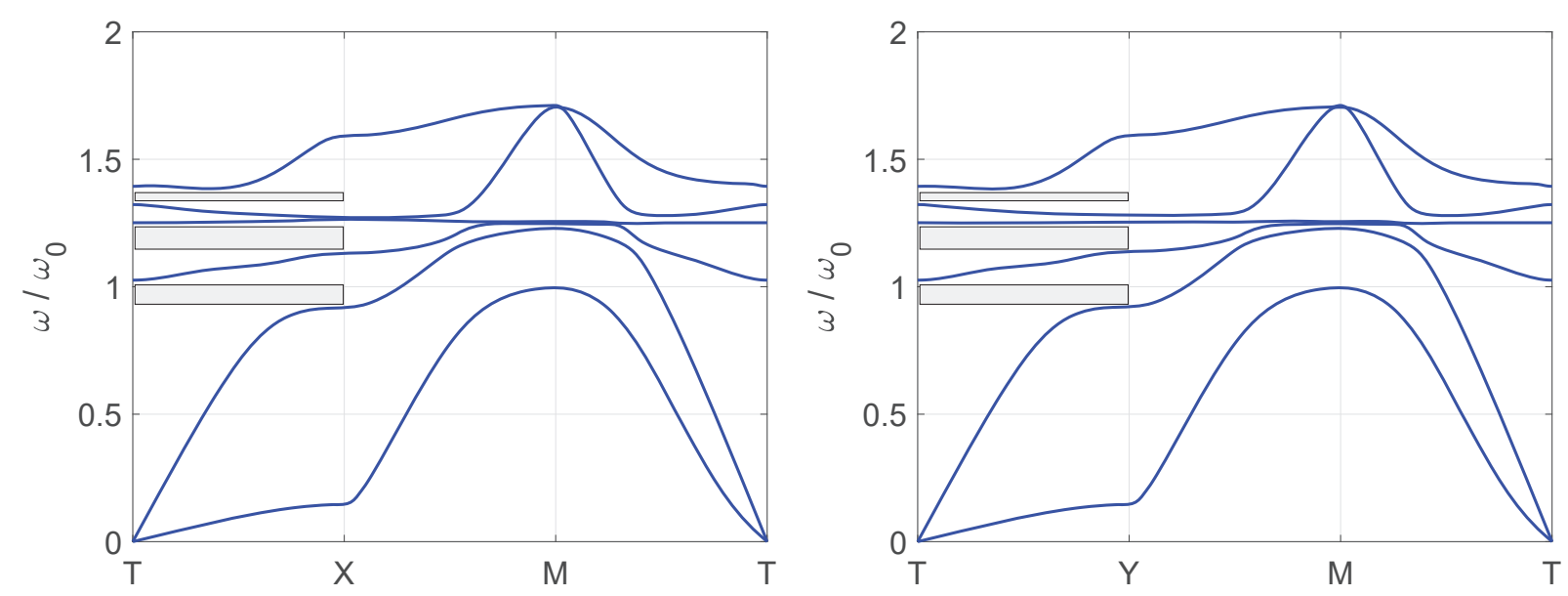

(b)

(c)

Figure 20: Bi-material auxetic micro-structure with $\nu *=-0.76\left(V_{f}=0.6, V_{h 1}=0.3\right): 3 \times 3$ array of cells (a) and band diagrams (b-c).

depicted in Figure 20(a). This micro-structure is a modification of that shown in Figure 15(a) that has been achieved for the distribution of the same amount of material $\left(V_{f}=0.6\right)$ and void. The bi-material micro-structure exhibits a stronger auxetic behavior, but the full band gap originally 


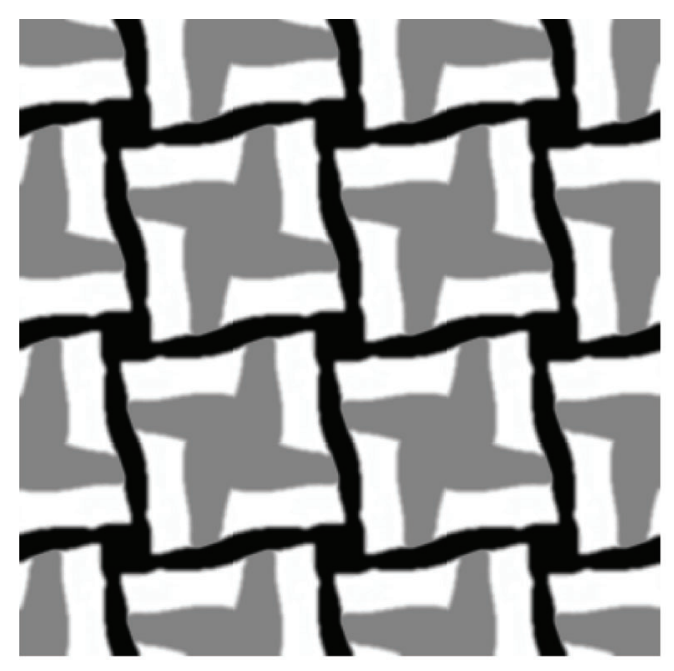

(a)
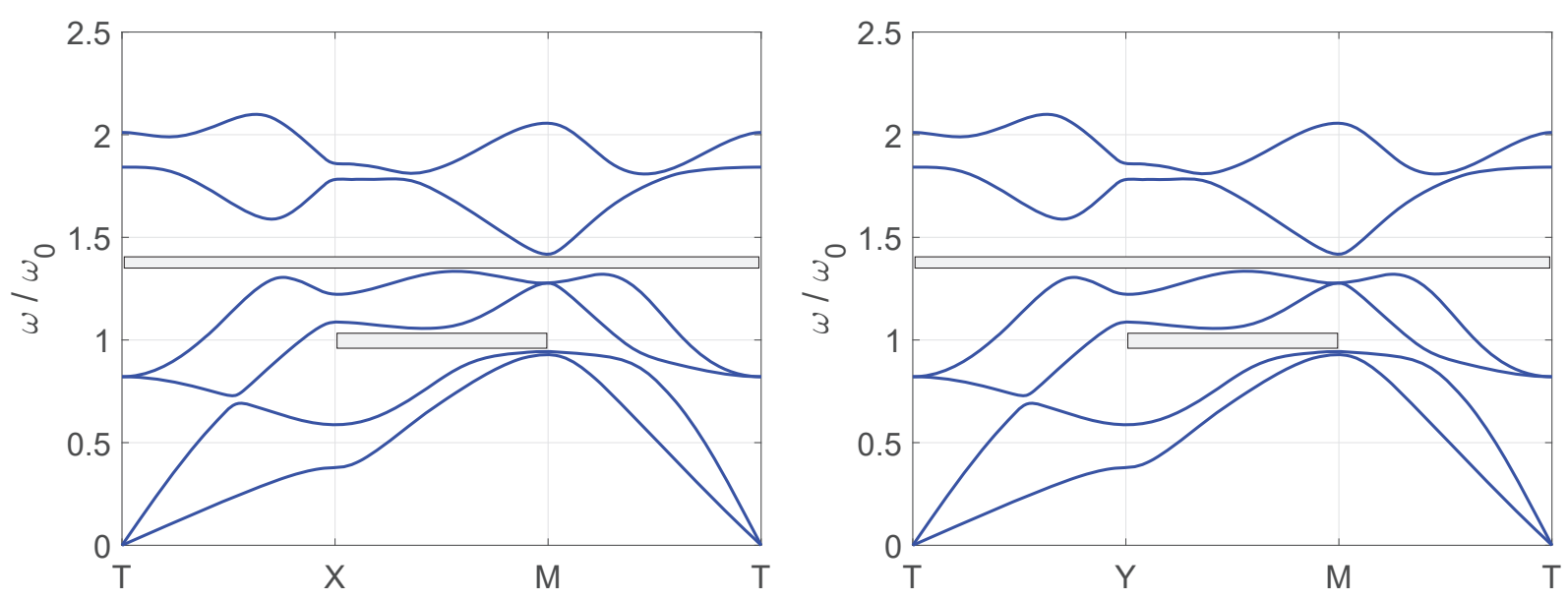

(b)

(c)

Figure 21: Bi-material auxetic micro-structure with $\nu *=-0.74\left(V_{f}=0.6, V_{h 1}=0.2\right): 3 \times 3$ array of cells (a) and band diagrams (b-c).

observed in Figure $15(\mathrm{~b}-\mathrm{c})$ is replaced by minor directional band gaps in $\Gamma-X / Y$, as similarly found for the previous multi-material periodic cells.

A different topology is represented in Figure 21(a), as found enforcing a maximum amount of 

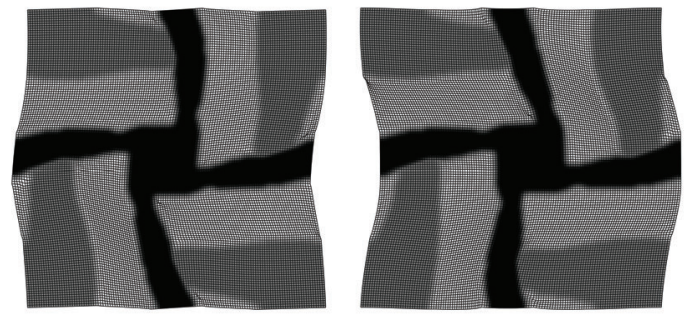

(a)

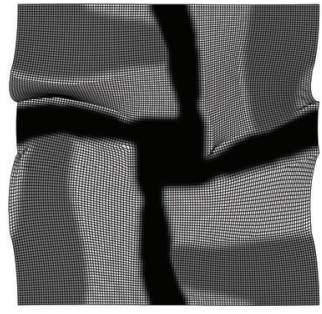

(b)

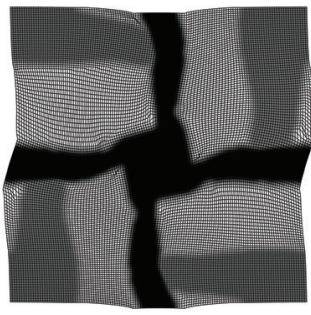

(c)

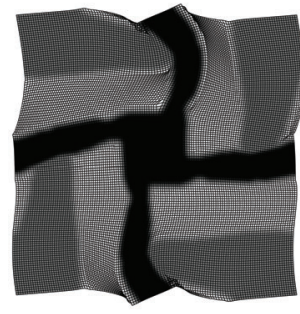

(d) (e)

Figure 22: Bi-material auxetic micro-structure with $\nu *=-0.74\left(V_{f}=0.6, V_{h 1}=0.2\right)$ : 1st (a), 2nd (b), 3rd (c), 4 th (d), 5 th (e) eigenmode for $k_{x}=0, k_{y}=\pi / \bar{y}(Y)$.

material $V_{f}=0.6$ with maximum volume of hard material $V_{h 1}=0.2$. The distribution of hard material is very similar to the layout shown in Figure 12. The soft material is arranged to provide tetrachiral inclusions connected to the eccentric arms of the chiral micro-structure made of hard material. This periodic layout has a full band gap between the 4th and the 5th eigenmode, see Figure 21(b-c). The 4th eigenmode is the first rotational mode at $Y$, see Figure 22.

A very similar topology can be found for the same volume fractions using combination 2 for the hard and soft material components. Figure 23(a) shows the achieved optimal layout. Notwithstanding the increased overall stiffness, see Table 2, the micro-structure exhibits the same equivalent negative Poisson's ratio. In this case, the band gap between the 4th and the 5th eigenmode is limited to wave vectors in the range $X / Y-M-\Gamma$, see Figure 23(b-c).

In the work by [59] an anisotropic periodic material made by core regions with ligaments inclined at a certain angle was found to exhibit stop bands associated to rotational modes of the localized resonators. Selective filtering properties of hexachiral honeycombs have been enhanced in [22] adopting inter-ring massive inclusions that play as oscillators elastically coupled to the micro-structure. Following these contributions, band gap properties of the topology in Figure 21 (or that in Figure 23) could be modified acting on the mechanical properties and the topology of 


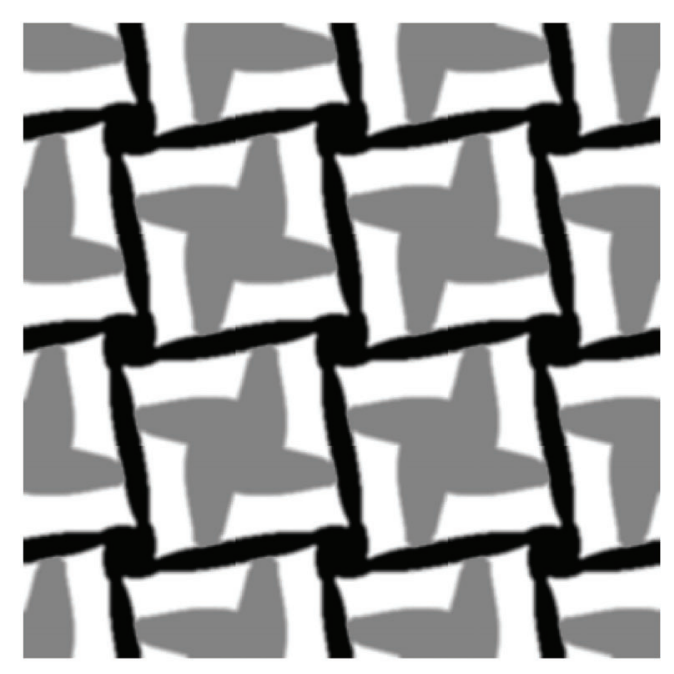

(a)
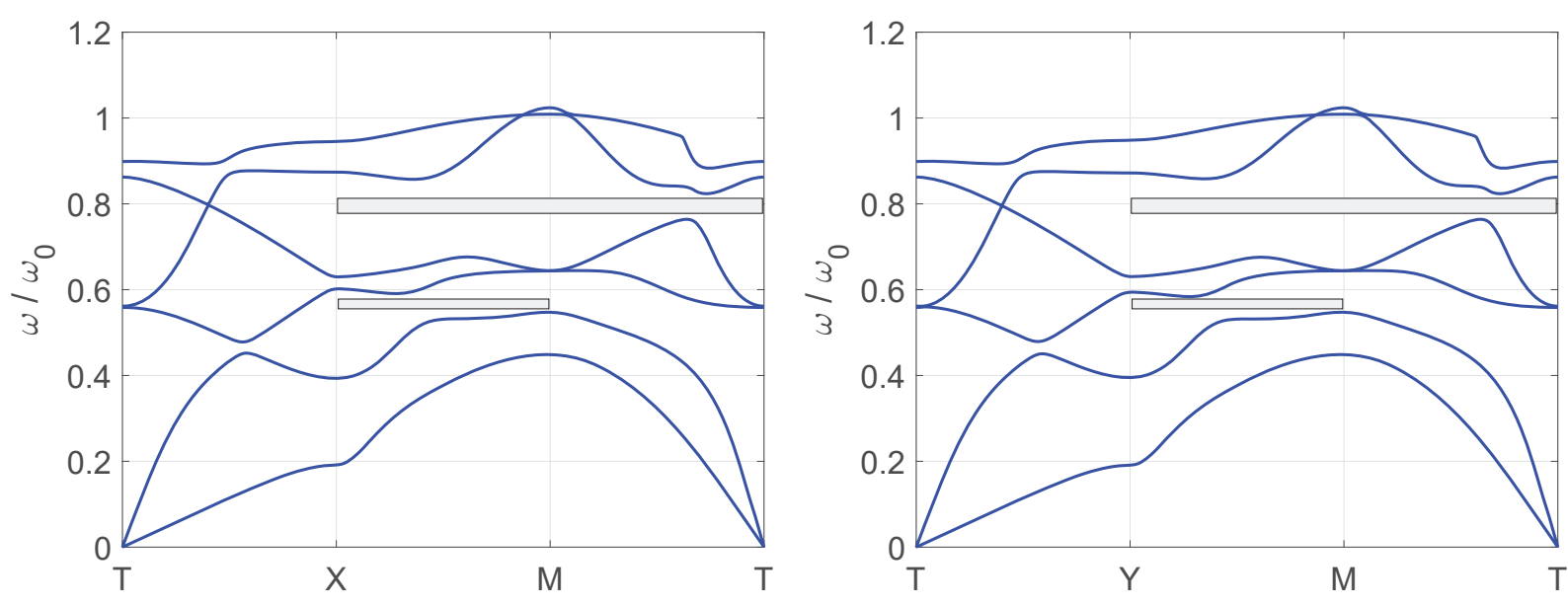

(b)

(c)

Figure 23: Bi-material auxetic micro-structure with $\nu *=-0.74\left(V_{f}=0.6, V_{h 2}=0.2\right): 3 \times 3$ array of cells (a) and band diagrams $(b-c)$.

the inclusions.

Figure 24(a) shows another topology that has been synthesized using combination 2 for the hard and soft material components. The equivalent Poisson's ratio is $\nu_{12}^{*}=\nu_{21}^{*}=\nu *=-0.78$, 


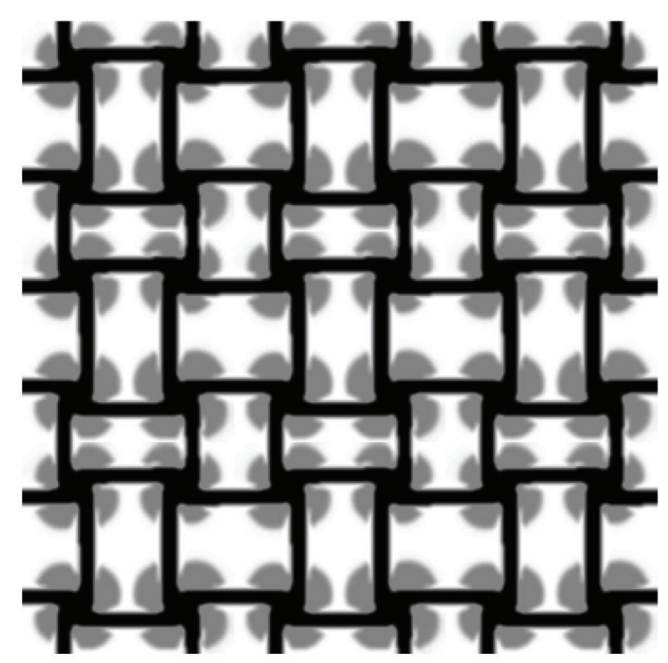

(a)
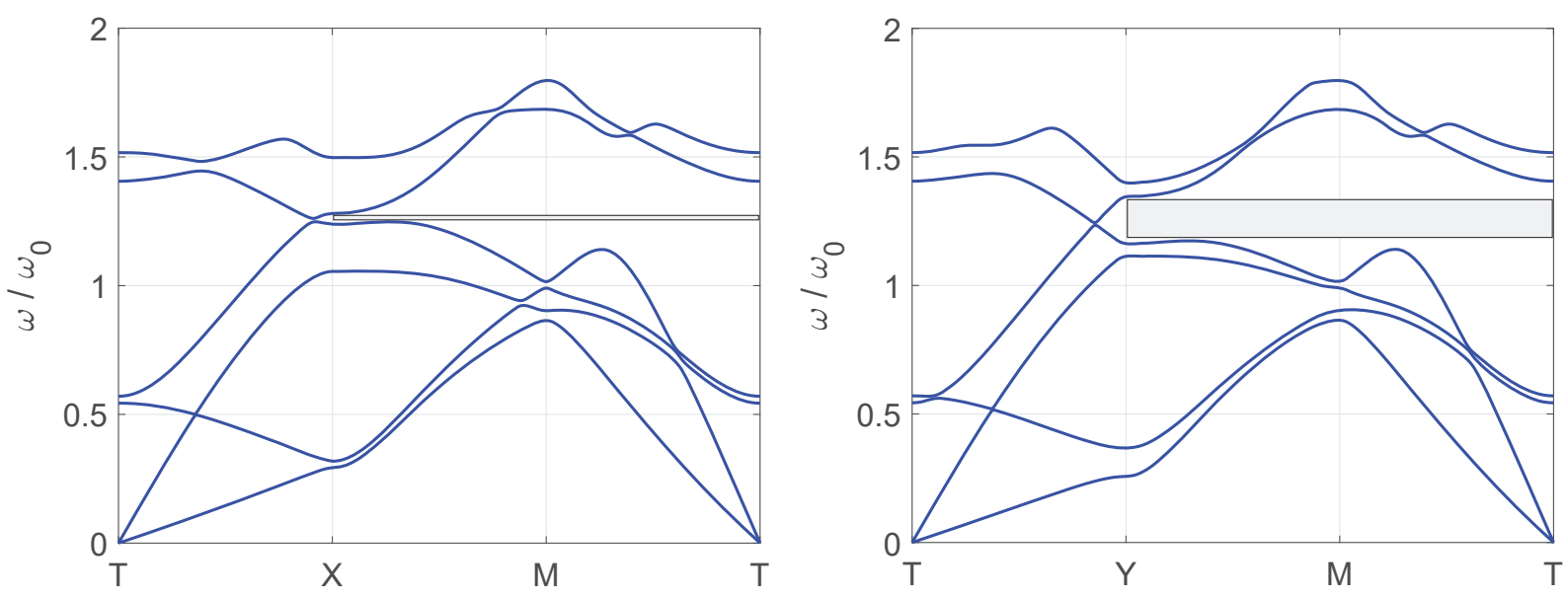

(b)

(c)

Figure 24: Bi-material auxetic micro-structure with $\nu *=-0.78\left(V_{f}=0.6, V_{h 2}=0.3\right): 3 \times 3$ array of cells (a) and band diagrams (b-c).

which is the minimum value found in the performed simulations considering distribution of two materials and void. The optimal layout is an anti-chiral tessellation of tetrachiral unit, resembling the single material design in Figure 7(a). Filtering properties are similar, since a directional band 
gap arises between the 4 -th and the 5 -th eigenmode for wave vectors in $X / Y-M-\Gamma$. In the achieved bi-material design, the soft phase surrounds the nodes of the anti-chiral network of hard material. This layout is quite similar to that considered in the work by [60] that addresses the optimal design of the frequency spectrum of anti-tetrachiral lattice metamaterials. Hence, a customization of the achieved layout for filtering purposes could be performed acting on the topology of the soft phase to provide local resonators to the micro-structure.

\section{Conclusions and perspectives}

A numerical study has been presented, addressing the design of two-dimensional auxetic periodic micro-structures by means of an inverse energy-based homogenization approach and investigating the band gap properties of the achieved layouts. SIMP has been used to distribute material and void, whereas a combined SIMP/RAMP scheme has been adopted to synthesize optimal bimaterial layouts. The density-based filter has been implemented in a volume-constrained optimization formulation whose objective function is conceived to achieve stiff micro-structures exhibiting the minimum equivalent Poisson's ratio. The arising optimization problem is highly non-convex and, solved through sequential convex programming for different sets of starting guesses, allows to explore several local minima with the same numerical tool. The same algorithm is able to find reentrant layouts, chiral and anti-chiral tessellation of tetra-chiral unit cells with material and void, exhibiting negative Poisson's ratios up to $\nu *=-0.80$. The topologies of some well-known auxetic honeycombs that were originally conceived or synthesized via alternative theoretical approaches (or different numerical methods of material design) have been retrieved in the simulations, see in particular the anti-chiral layouts in Section 4.2 and some of the chiral layouts in Section 4.3. Other variations of auxetic patterns have been generated as well, see the re-entrant micro-structures of Section 4.1 and solutions from Section 4.4. Optimal bi-material layouts may consist of cells where the hard material acts as a coating for the soft one to provide rigid sub-structures that maxi- 
mize the auxetic deformation through suitable configurations. In other layouts, the soft material is arranged to provide additional auxetic shapes or localized inclusions within the main periodic micro-structure made of hard material.

As shown in [25] and discussed in Section 1, the auxetic feature can be conveniently exploited to synthesise tunable phononic crystals and wave guides. Hence, a Floquet-Bloch approach has been implemented to investigate possible band gaps characterizing the in-plane wave propagation in the achieved auxetic cells and identify types of micro-structures that could be effectively exploited for such kind of applications. A greater efficiency in band gap tuning in negative Poisson's ratio materials and combined optimization remain to be demonstrated, and this work is only a first exploratory step in this direction. Directional and full band-pass filters have been found in the case of micro-structures whose auxetic behavior comes from the arising of a rotational deformation of the periodic cell. The mode corresponding to the lower bound of any stop band has been shown to be rotational or related to deformations of the core within the (chiral) basic unit. In some of the achieved optimal bi-material layouts, the arrangement of the soft phase recalls local resonators that have been used in the literature to enhance the selective filtering properties of traditional chiral and anti-chiral honeycomb systems made of stiff disks and ligaments.

Numerical simulations on single-material design show that the band gap properties of similar layouts are remarkably affected by the volume fraction. The two anti-chiral tessellations of Section 4.2 have a very similar topology but use a different amount of material. It is found that their directional band gaps are different, both in terms of extension and central frequency. Similarly, the four-arm cell of Section 4.3 exhibits full or partial band gap depending on the volume fraction. This suggests the adoption of the volume fraction as a parameter to customize the dynamic properties of the auxetic layouts exhibiting band gap. The capabilities of the adopted minimizer to cope with multi-constrained problems could be exploited to enhance band gaps. Hence, the optimal design of tunable mechanical filters and phononic crystals could be handled through an extension of the 
proposed formulation for maximum auxeticity including constraints on the frequency spectrum and adopting the micro-structures achieved in this study as starting guesses of the optimization procedure.

Concerning multi-material design, stiffness and mass of the constituent phases affect not only the extension and the central frequency of band gaps. Two similar layouts are shown in Section 4.4 for which the arising of a full band gap depends on the adopted set of materials. In this regard, the proposed algorithm is an efficient numerical tool to explore solutions for several combinations of constituent phases and volume fractions, searching for minima of the non-convex objective function by means of a simple multi-start procedure. As already outlined for single-material design, enforcements on the frequency spectrum could be handled implementing a multi-constrained minimization that uses the achieved layouts as starting guesses. This point is currently under investigation.

The ongoing research is also devoted to the extension of the proposed procedure to the threedimensional framework, to explore 3D optimal auxetic solutions exhibiting band gap and address realistic applications of tunable wave-guiding / insulation. Indeed, the minimization problem herein considered is straightforward to be implemented in three dimensions, through a generalization of the energy-based homogenization approach and of the considered objective function. The bottle-neck of the approach would consist in the finite element analysis recursive task. Optimal layouts on fine discretizations would call for a parallelization of the code, whereas preliminary results on coarse meshes could be derived through a standard implementation. 
Appendix A. In-plane wave propagation in a linear elastic medium: effective stiffness matrices

The effective stiffness matrix $\mathbf{K}\left(\mathbf{k}, \mathbf{x}, \mathbf{x}_{\mathbf{e}}\right)$ of Eqn.(13) is gathered from the element-wise matrices $\mathbf{K}_{\mathbf{e}}$ as:

$$
\mathbf{K}_{\mathbf{e}}=\mathbf{K}_{0, e}-i\left(k_{1} \mathbf{K}_{1, e}+k_{2} \mathbf{K}_{2, e}\right)+k_{1}^{2} \mathbf{K}_{3, e}+k_{1} k_{2} \mathbf{K}_{4, e}+k_{2}^{2} \mathbf{K}_{5, e}
$$

whose components are herein reported for the sake of completeness:

$$
\begin{aligned}
& \mathbf{K}_{1, e}=\int_{\Omega_{e}} \mathbf{N}^{T} \boldsymbol{\alpha}_{1}^{T} \mathbf{C}_{\mathbf{e}}\left(x_{e}, x_{m, e}\right) \mathbf{B}-\left(\mathbf{N}^{T} \boldsymbol{\alpha}_{1}^{T} \mathbf{C}_{\mathbf{e}}\left(x_{e}, x_{m, e}\right) \mathbf{B}\right)^{T} d \Omega_{e}, \\
& \mathbf{K}_{2, e}=\int_{\Omega_{e}}^{\mathbf{N}^{T}} \boldsymbol{\alpha}_{2}^{T} \mathbf{C}_{\mathbf{e}}\left(x_{e}, x_{m, e}\right) \mathbf{B}-\left(\mathbf{N}^{T} \boldsymbol{\alpha}_{2}^{T} \mathbf{C}_{\mathbf{e}}\left(x_{e}, x_{m, e}\right) \mathbf{B}\right)^{T} d \Omega_{e}, \\
& \mathbf{K}_{3, e}=\int_{\Omega_{e}}^{\mathbf{N}^{T}} \boldsymbol{\alpha}_{1}^{T} \mathbf{C}_{\mathbf{e}}\left(x_{e}, x_{m, e}\right) \boldsymbol{\alpha}_{1} \mathbf{N} d \Omega_{e} \\
& \mathbf{K}_{4, e}=\int_{\Omega_{e}}^{\mathbf{N}^{T}}\left(\boldsymbol{\alpha}_{1}^{T} \mathbf{C}_{\mathbf{e}}\left(x_{e}, x_{m, e}\right) \boldsymbol{\alpha}_{2}+\left(\boldsymbol{\alpha}_{1}^{T} \mathbf{C}_{\mathbf{e}}\left(x_{e}, x_{m, e}\right) \boldsymbol{\alpha}_{2}\right)^{T}\right) \mathbf{N} d \Omega_{e}, \\
& \mathbf{K}_{5, e}=\int_{\Omega_{e}}^{\mathbf{N}^{T}} \boldsymbol{\alpha}_{2}^{T} \mathbf{C}_{\mathbf{e}}\left(x_{e}, x_{m, e}\right) \boldsymbol{\alpha}_{2} \mathbf{N} d \Omega_{e}
\end{aligned}
$$

\section{Acknowledgement}

The authors gratefully acknowledge the financial support of the Italian Ministry of Education University and Research through grant number PRIN15-2015LYYXA8 for the project Multi-scale mechanical models for the design and optimization of micro-structured smart materials and metamaterials.

Funding: This study was funded by MIUR Italian Ministry of Education University and Research (grant number PRIN15-2015LYYXA8).

Conflict of Interest: The authors declare that they have no conflict of interest 
[1] Kshetrimayum, R. S. (2004). A brief intro to metamaterials. IEEE Potentials, 23(5), 44-46. doi:10.1109/MP.2005.1368916

[2] Choi, J. B., Lakes, R. S. (1992). Non-linear properties of metallic cellular materials with a negative poisson's ratio. Journal of Materials Science, 27(19), 5375-5381. doi:10.1007/BF02403846

[3] Argatov, I. I., Guinovart-Díaz, R., Sabina, F. J. (2012). On local indentation and impact compliance of isotropic auxetic materials from the continuum mechanics viewpoint. International Journal of Engineering Science, 54, 42-57. doi:10.1016/j.ijengsci.2012.01.010

[4] Choi, J. B., Lakes, R. S. (1996). Fracture toughness of re-entrant foam materials with a negative poisson's ratio: Experiment and analysis. International Journal of Fracture, 80(1), 73-83. doi:10.1007/BF00036481

[5] Lakes, R. (1987). Foam structures with a negative poisson's ratio. Science, 235(4792), 10381040. doi:10.1126/science.235.4792.1038

[6] Alderson, A., Rasburn, J., Ameer-Beg, S., Mullarkey, P. G., Perrie, W., Evans, K. E. (2000). An auxetic filter: A tuneable filter displaying enhanced size selectivity or defouling properties. Industrial and Engineering Chemistry Research, 39(3), 654-665. doi:10.1021/ie990572w

[7] Imbalzano, G., Tran, P., Ngo, T. D., Lee, P. V. S. (2016). A numerical study of auxetic composite panels under blast loadings. Composite Structures, 135, 339-352. doi:10.1016/j.compstruct.2015.09.038

[8] Evans, K. E., Nkansah, M. A., Hutchinson, I. J., Rogers, S. C. (1991). Molecular network design. Nature, 353(6340), 124. doi:10.1038/353124a0 
[9] Ren, X., Das, R., Tran, P., Ngo, T. D., Xie, Y. M. (2018). Auxetic metamaterials and structures: A review. Smart Materials and Structures, 27(2) doi:10.1088/1361-665X/aaa61c

[10] Grima, J. N., Farrugia, P., Gatt, R., Attard, D. (2008). On the auxetic properties of rotating rhombi and parallelograms: A preliminary investigation. Physica Status Solidi (B) Basic Research, 245(3), 521-529. doi:10.1002/pssb.200777705

[11] Grima, J. N., Winczewski, S., Mizzi, L., Grech, M. C., Cauchi, R., Gatt, R., Attard, D., Wojciechowski K.W., Rybicki, J. (2015). Tailoring graphene to achieve negative poisson's ratio properties. Advanced Materials, 27(8), 1455-1459. doi:10.1002/adma.201404106

[12] Shan, S., Kang, S. H., Zhao, Z., Fang, L., Bertoldi, K. (2015). Design of planar isotropic negative poisson's ratio structures. Extreme Mechanics Letters, 4, 96-102. doi:10.1016/j.eml.2015.05.002

[13] Carta, G., Brun, M., Baldi, A. (2016). Design of a porous material with isotropic negative poisson's ratio. Mechanics of Materials, 97, 67-75. doi:10.1016/j.mechmat.2016.02.012

[14] Gibson, L. J., Ashby, M. F., Schajer, G. S., Robertson, C. I. (1982). Mechanics of twodimensional cellular materials. Proceedings of the Royal Society of London, Series A: Mathematical and Physical Sciences, 382(1782), 25-42

[15] Lakes, R. (1991). Deformation mechanisms in negative poisson's ratio materials: Structural aspects. Journal of Materials Science, 26(9), 2287-2292. doi:10.1007/BF01130170

[16] Brillouin, L. (1953) Wave Propagation in Periodic Structures, 2nd Edn. New York: Dover

[17] Hussein, M. I., Hulbert, G. M., Scott, R. A. (2003). Band-gap engineering of elastic waveguides using periodic materials. Paper presented at the American Society of Mechanical Engineers, Design Engineering Division (Publication) DE, , 116(2) 799-807. doi:10.1115/IMECE2003-41886 
[18] Sigmund, O., Jensen, J. S. (2003). Systematic design of phononic band-gap materials and structures by topology optimization. Philosophical Transactions of the Royal Society A: Mathematical, Physical and Engineering Sciences, 361(1806), 1001-1019. doi:10.1098/rsta.2003.1177

[19] Martinsson, P.G., Movchan, A.B. (2003). Vibrations of lattice structures and phononic band gaps. Quarterly Journal of Mechanics and Applied Mathematics, 56 (1), 45-64. doi: 10.1093/qj$\operatorname{mam} / 56.1 .45$

[20] Ruzzene, M., Scarpa, F. (2005). Directional and band-gap behavior of periodic auxetic lattices. Physica Status Solidi (B) Basic Research, 242(3), 665-680. doi:10.1002/pssb.200460385

[21] Billon, K., Zampetakis, I., Scarpa, F., Ouisse, M., Sadoulet-Reboul, E., Collet, M., Perriman, A., Hetherington, A. (2017). Mechanics and band gaps in hierarchical auxetic rectangular perforated composite metamaterials. Composite Structures, 160, 1042-1050. doi:10.1016/j.compstruct.2016.10.121

[22] Bacigalupo, A., Lepidi, M., Gnecco, G., Gambarotta, L. (2016). Optimal design of auxetic hexachiral metamaterials with local resonators. Smart Materials and Structures, 25(5) doi:10.1088/0964-1726/25/5/054009

[23] D’Alessandro, L., Belloni, E., Ardito, R., Corigliano, A., Braghin, F. (2016). Modeling and experimental verification of an ultra-wide bandgap in 3D phononic crystal. Applied Physics Letters, 109(22) doi:10.1063/1.4971290

[24] D’Alessandro, L., Belloni, E., Ardito, R., Braghin, F., Corigliano, A. (2017). Mechanical lowfrequency filter via modes separation in 3D periodic structures. Applied Physics Letters, 111(23) doi:10.1063/1.4995554

[25] D’Alessandro, L., Zega, V., Ardito, R., Corigliano, A. (2018). 3D auxetic single material 
periodic structure with ultra-wide tunable bandgap. Scientific Reports, 8(1) doi:10.1038/s41598018-19963-1

[26] Krödel, S., Delpero, T., Bergamini, A., Ermanni, P., Kochmann, D. M. (2014). 3D auxetic microlattices with independently controllable acoustic band gaps and quasi-static elastic moduli. Advanced Engineering Materials, 16(4), 357-363. doi:10.1002/adem.201300264

[27] Bendsøe, M. P., Kikuchi, N. (1988). Generating optimal topologies in structural design using a homogenization method. Computer Methods in Applied Mechanics and Engineering, 71(2), 197-224. doi:10.1016/0045-7825(88)90086-2

[28] Sigmund, O. (1994). Materials with prescribed constitutive parameters: An inverse homogenization problem. International Journal of Solids and Structures, 31(17), 2313-2329. doi:10.1016/0020-7683(94)90154-6

[29] Sigmund, O., Torquato, S. (1999). Design of smart composite materials using topology optimization. Smart Materials and Structures, 8(3), 365-379. doi:10.1088/0964-1726/8/3/308

[30] Osanov, M., Guest, J. K. (2016). Topology optimization for architected materials design doi:10.1146/annurev-matsci-070115-031826

[31] Allaire, G., De Gournay, F., Jouve, F., Toader, A. -. (2005). Structural optimization using topological and shape sensitivity via a level set method. Control and Cybernetics, 34(1), 59-81.

[32] Wang, M. Y., Wang, X. (2005). A level-set based variational method for design and optimization of heterogeneous objects. CAD Computer Aided Design, 37(3), 321-337. doi:10.1016/j.cad.2004.03.007

[33] Amstutz, S., Giusti, S. M., Novotny, A. A., De Souza Neto, E. A. (2010). Topological deriva- 
tive for multi-scale linear elasticity models applied to the synthesis of micro-structures. International Journal for Numerical Methods in Engineering, 84(6), 733-756. doi:10.1002/nme.2922

[34] Bruggi, M., Zega, V., Corigliano, A. (2017). Synthesis of auxetic structures using optimization of compliant mechanisms and a micropolar material model. Structural and Multidisciplinary Optimization, 55(1) doi:10.1007/s00158-016-1589-9

[35] Xia, L., Breitkopf, P.(2015) Design of materials using topology optimization and energy-based homogenization approach in Matlab, Structural and Multidisciplinary Optimization 52(6), 12291241

[36] Andreassen, E., Lazarov, B. S., Sigmund, O. (2014). Design of manufacturable 3D extremal elastic micro-structure. Mechanics of Materials, 69(1), 1-10. doi:10.1016/j.mechmat.2013.09.018

[37] Clausen, A., Wang, F., Jensen, J. S., Sigmund, O., Lewis, J. A. (2015). Topology optimized architectures with programmable poisson's ratio over large deformations. Advanced Materials, 27(37), 5523-5527. doi:10.1002/adma.201502485

[38] Vogiatzis, P., Chen, S., Wang, X., Li, T., Wang, L. (2017). Topology optimization of multimaterial negative poisson's ratio metamaterials using a reconciled level set method. CAD Computer Aided Design, 83, 15-32. doi:10.1016/j.cad.2016.09.009

[39] Zhang, H., Luo, Y., Kang, Z. (2018). Bi-material microstructural design of chiral auxetic metamaterials using topology optimization. Composite Structures, 195, 232-248. doi:10.1016/j.compstruct.2018.04.058

[40] Schwerdtfeger, J., Wein, F., Leugering, G., Singer, R. F., Körner, C., Stingl, M., Schury, F. (2011). Design of auxetic structures via mathematical optimization. Advanced Materials, 23(22-23), 2650-2654. doi:10.1002/adma.201004090 
[41] Zong, H., Zhang, H., Wang, Y., Wang, M. Y., Fuh, J. Y. H. (2018). On two-step design of micro-structure with desired poisson's ratio for AM. Materials and Design, 159, 90-102. doi:10.1016/j.matdes.2018.08.032

[42] Stolpe, M., Svanberg, K. (2001). An alternative interpolation scheme for minimum compliance topology optimization. Structural and Multidisciplinary Optimization, 22(2), 116-124. doi:10.1007/s001580100129

[43] Svanberg, K. (1987). The method of moving asymptotes - a new method for structural optimization. International Journal for Numerical Methods in Engineering, 24(2), 359-373. doi:10.1002/nme.1620240207

[44] Kittel, C. (1986) Solid State Physics. Wiley

[45] Guedes, J., Kikuchi, N. (1990). Preprocessing and postprocessing for materials based on the homogenization method with adaptive finite element methods, Computer Methods in Applied Mechanics and Engineering 83(2), 143-198.

[46] Bensoussan, A., Lions, J.L., Papanicolaou, G. (1978) Asymptotic Analysis for Periodic Structures. North-Holland

[47] Evans, K.E., 1991. Auxetic polymers: a new range of materials. Endeavour 15 (4), 170-174. doi:10.1016/0160-9327(91)90123-S

[48] Collet, M., Noël, L., Bruggi, M., Duysinx, P. (2018). Topology optimization for microstructural design under stress constraints. Structural and Multidisciplinary Optimization, 58(6), 2677-2695. doi:10.1007/s00158-018-2045-9

[49] Bourdin B (2001) Filters in topology optimization. Int J Numer Methods Eng 50(9):2143-2158 
[50] Mathews, J., Walker, R. (1964) Mathematical Methods of Physics. Addison-Wesley

[51] Andreassen, E., Jensen, J. S. (2013). Analysis of phononic bandgap structures with dissipation. Journal of Vibration and Acoustics, Transactions of the ASME, 135(4) doi:10.1115/1.4023901

[52] Wang, Y., Wang, Y., Zhang, C. (2014). Bandgaps and directional propagation of elastic waves in 2D square zigzag lattice structures. Journal of Physics D: Applied Physics, 47(48) doi:10.1088/0022-3727/47/48/485102

[53] Zega, V., Credi, C., Bernasconi, R., Langfelder, G., Magagnin, L., Levi, M., Corigliano, A. (2018). The first 3-d-printed z-axis accelerometers with differential capacitive sensing. IEEE Sensors Journal, 18(1), 53-60. doi:10.1109/JSEN.2017.2768299

[54] Sigmund, O., Torquato, S., Aksay, I. A. (1998). On the design of 1-3 piezocomposites using topology optimization. Journal of Materials Research, 13(4), 1038-1048. doi:10.1557/JMR.1998.0145

[55] Grima, J. N., Gatt, R., Farrugia, P. (2008). On the properties of auxetic meta-tetrachiral structures. Physica Status Solidi (B) Basic Research, 245(3), 511-520. doi:10.1002/pssb.200777704

[56] Smith, C. W., Grima, J. N., Evans, K. E. (2000). Novel mechanism for generating auxetic behaviour in reticulated foams: Missing rib foam model. Acta Materialia, 48(17), 4349-4356. doi:10.1016/S1359-6454(00)00269-X

[57] Wang, F., Sigmund, O., Jensen, J. S. (2014). Design of materials with prescribed nonlinear properties. Journal of the Mechanics and Physics of Solids, 69(1), 156-174. doi:10.1016/j.jmps.2014.05.003

[58] Spadoni, A., Ruzzene, M., Gonella, S., Scarpa, F. (2009). Phononic properties of hexagonal chiral lattices. Wave Motion, 46(7), 435-450. doi:10.1016/j.wavemoti.2009.04.002 
[59] Bigoni, D., Guenneau, S., Movchan, A. B., Brun, M. (2013). Elastic metamaterials with inertial locally resonant structures: Application to lensing and localization. Physical Review B - Condensed Matter and Materials Physics, 87(17) doi:10.1103/PhysRevB.87.174303

[60] Bacigalupo, A., Gnecco, G., Lepidi, M., Gambarotta, L. (2017). Optimal design of lowfrequency band gaps in anti-tetrachiral lattice meta-materials. Composites Part B: Engineering, 115, 341-359. doi:10.1016/j.compositesb.2016.09.062 


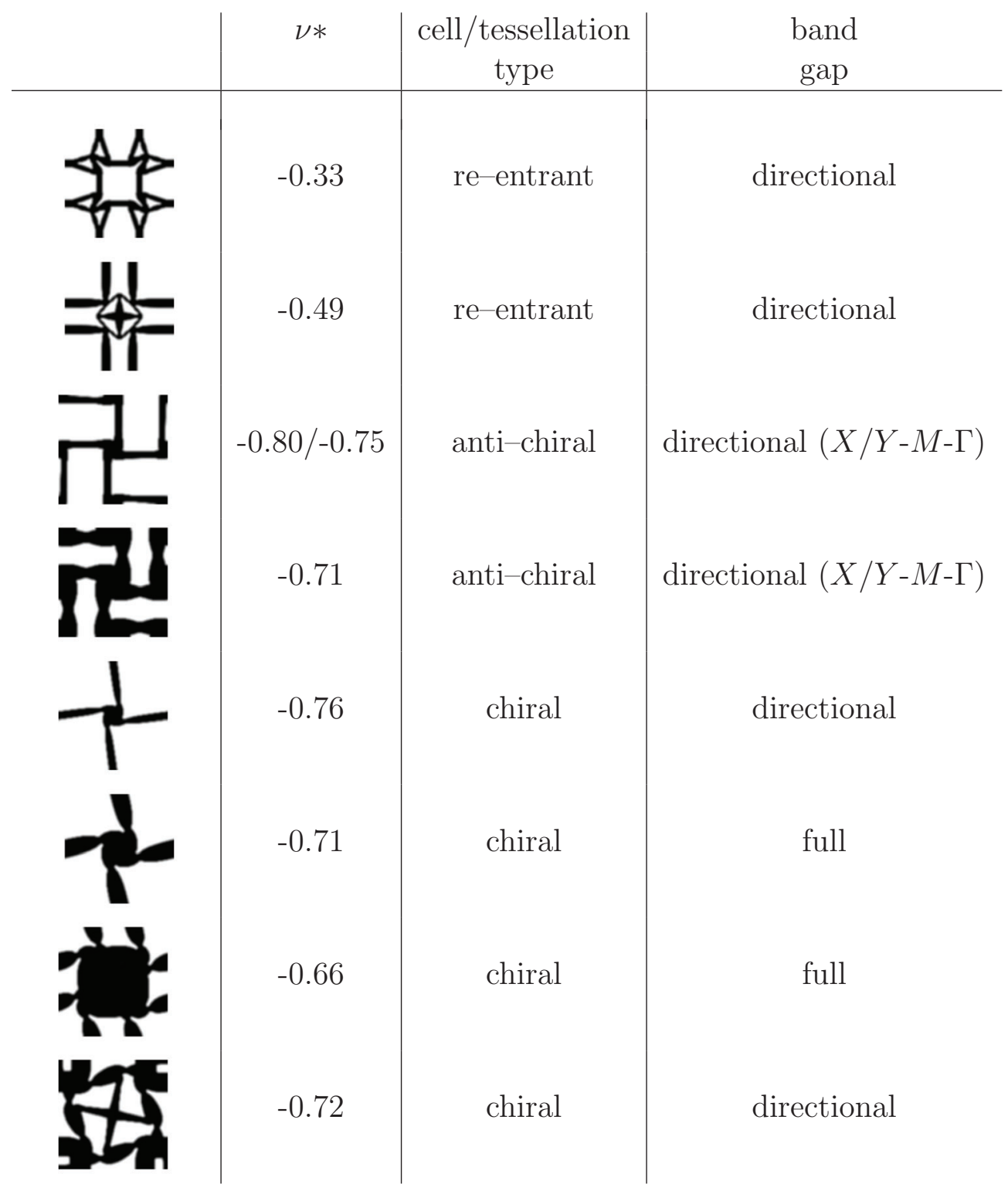

Table 3: Overview of the optimal micro-structures achieved by distribution of material and void: topology of the unit cell, effective Poisson's ratio(s), cell/tessellation type and band gaps. 


\begin{tabular}{c|c|c|c}
$\nu *$ & $\begin{array}{c}\text { cell/tessellation } \\
\text { type }\end{array}$ & $\begin{array}{c}\text { band } \\
\text { gap }\end{array}$ \\
\hline & -0.69 & chiral & directional \\
\hline
\end{tabular}

Table 4: Overview of the optimal micro-structures achieved by distribution of two materials and void: topology of the unit cell, effective Poisson's ratio, cell/tessellation type and band gaps. 